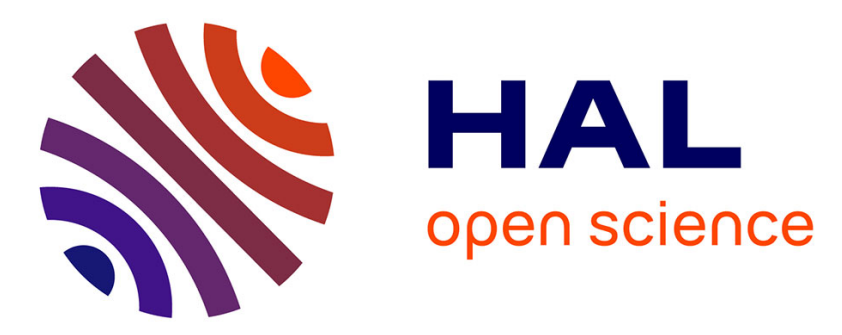

\title{
DECAY OF SUPERCONDUCTIVITY AWAY FROM THE MAGNETIC ZERO SET
}

\author{
Bernard Helffer, Ayman Kachmar
}

\section{To cite this version:}

Bernard Helffer, Ayman Kachmar. DECAY OF SUPERCONDUCTIVITY AWAY FROM THE MAG-

NETIC ZERO SET. Calculus of Variations and Partial Differential Equations, 2017, 56. hal-01435147

\section{HAL Id: hal-01435147 \\ https://hal.science/hal-01435147}

Submitted on 13 Jan 2017

HAL is a multi-disciplinary open access archive for the deposit and dissemination of scientific research documents, whether they are published or not. The documents may come from teaching and research institutions in France or abroad, or from public or private research centers.
L'archive ouverte pluridisciplinaire HAL, est destinée au dépôt et à la diffusion de documents scientifiques de niveau recherche, publiés ou non, émanant des établissements d'enseignement et de recherche français ou étrangers, des laboratoires publics ou privés. 


\title{
DECAY OF SUPERCONDUCTIVITY AWAY FROM THE MAGNETIC ZERO SET
}

\author{
BERNARD HELFFER AND AYMAN KACHMAR
}

\begin{abstract}
We establish exponential bounds on the Ginzburg-Landau order parameter away from the curve where the applied magnetic field vanishes. In the units used in this paper, the estimates are valid when the parameter measuring the strength of the applied magnetic field is comparable with the Ginzburg-Landau parameter. This completes a previous work by the authors analyzing the case when this strength was much higher. Our results display the distribution of surface and bulk superconductivity and are valid under the assumption that the magnetic field is Hölder continuous.
\end{abstract}

\section{INTRODUCTION}

1.1. The functional. In non-dimensional units, the Ginzburg-Landau functional is defined as follows,

$$
\mathcal{E}(\psi, \mathbf{A})=\int_{\Omega}\left(|(\nabla-i \kappa H \mathbf{A}) \psi|^{2}-\kappa^{2}|\psi|^{2}+\frac{\kappa^{2}}{2}|\psi|^{4}+(\kappa H)^{2}\left|\operatorname{curl} \mathbf{A}-B_{0}\right|^{2}\right) d x,
$$

where:

- $\Omega \subset \mathbb{R}^{2}$ is an open, bounded and simply connected set with a $C^{\infty}$ boundary;

- $(\psi, \mathbf{A}) \in H^{1}(\Omega ; \mathbb{C}) \times H^{1}\left(\Omega ; \mathbb{R}^{2}\right)$;

- $\kappa>0$ and $H>0$ are two parameters ;

- $B_{0}$ is a real-valued function in $L^{2}(\Omega)$.

The superconducting sample is supposed to occupy a long cylinder with vertical axis and horizontal cross section $\Omega$. The parameter $\kappa$ is the Ginzburg-Landau parameter that expresses the properties of the superconducting material. The applied magnetic field is $\kappa H B_{0} \vec{e}$, where $\vec{e}=(0,0,1)$. The configuration pair $(\psi, \mathbf{A})$ describes the state of superconductivity as follows: $|\psi|^{2}$ measures the density of the superconducting Cooper pairs, curl A measures the induced magnetic field in the sample and $j:=(i \psi, \nabla \psi-i \kappa H \mathbf{A} \psi)$ measures the induced super-current. Here $(\cdot, \cdot)$ denotes the inner product in $\mathbb{C}$ defined as follows, $(u, v)=u_{1} v_{1}+u_{2} v_{2}$ where $u=u_{1}+i u_{2}$ and $v=v_{1}+i v_{2}$.

At equilibrium, the state of the superconductor is described by the (minimizing) configurations $(\psi, \mathbf{A})$ that realize the following ground state energy

$$
\mathrm{E}_{\mathrm{gs}}(\kappa, H)=\inf \left\{\mathcal{E}(\psi, \mathbf{A}):(\psi, \mathbf{A}) \in H^{1}(\Omega ; \mathbb{C}) \times H^{1}\left(\Omega ; \mathbb{R}^{2}\right)\right\} .
$$

Such configurations are critical points of the functional introduced in (1.1), that is they solve the following system of Euler-Lagrange equations ( $\nu$ is the unit inward normal on the boundary)

$$
\left\{\begin{aligned}
-(\nabla-i \kappa H \mathbf{A})^{2} \psi & =\kappa^{2}\left(1-|\psi|^{2}\right) \psi & & \text { in } \Omega, \\
-\nabla^{\perp}\left(\operatorname{curl} \mathbf{A}-B_{0}\right) & =(\kappa H)^{-1} \operatorname{Im}(\bar{\psi}(\nabla-i \kappa H \mathbf{A}) \psi) & & \text { in } \Omega, \\
\nu \cdot(\nabla-i \kappa H \mathbf{A}) \psi & =0 & & \text { on } \partial \Omega \\
\operatorname{curl} \mathbf{A} & =B_{0} & & \text { on } \partial \Omega .
\end{aligned}\right.
$$

Once a choice of $(\kappa, H)$ is fixed, the notation $(\psi, \mathbf{A})_{\kappa, H}$ stands for a solution of (1.3). When $B_{0}$ belongs to $C^{0}(\bar{\Omega})$, we introduce two constants $\beta_{0}$ and $\beta_{1}$ that will play a central role in this 
paper:

$$
\beta_{0}:=\sup _{x \in \bar{\Omega}}\left|B_{0}(x)\right| \quad \text { and } \quad \beta_{1}:=\sup _{x \in \partial \Omega}\left|B_{0}(x)\right| .
$$

1.2. The case with a constant magnetic. A huge mathematical literature is devoted to the analysis of the functional in (1.1) when the magnetic field is constant. This corresponds to taking $B_{0}=1$ in (1.1). The two monographs $[15,39]$ and the references therein are mainly devoted to this subject. One important situation is the transition from bulk to surface superconductivity. This happens when the parameter $H$ increases between two critical values $H_{C_{2}}$ and $H_{C_{3}}$ called the second and third critical fields respectively.

In this analysis the de Gennes constant plays a central role. This constant is universal and defined as follows

$$
\Theta_{0}=\inf _{\xi \in \mathbb{R}}\left\{\inf _{\|u\|_{2}=1}\left(\int_{0}^{\infty}\left(\left|u^{\prime}(t)\right|^{2}+(t-\xi)^{2}|u(t)|^{2}\right) d t\right)\right\} .
$$

Furthermore, it is known (cf. [15]) that

$$
\frac{1}{2}<\Theta_{0}<1
$$

The de Gennes constant appears indeed in the asymptotics of $H_{C_{3}}$ for $\kappa$ large

$$
H_{C_{3}} \sim \Theta_{0}^{-1} \kappa,
$$

while we have for the second critical field

$$
H_{C_{2}} \sim \kappa
$$

To be more specific, if $b>0$ is a constant and $(\psi, \mathbf{A})_{\kappa, H}$ is a minimizer of the functional in (1.1) for $H=b \kappa$ (and $B_{0}=1$ ), the concentration of $\psi$ in the limit $\kappa \rightarrow \infty$ depends strongly on $b$.

If $0<b<1$, then $\psi$ is uniformly distributed in the domain $\Omega$ (cf. [28, 40]). If $1<b<\Theta_{0}^{-1}$, then $\psi$ is concentrated on the surface and decays exponentially in the bulk (cf. [12, 34]). If $b>\Theta_{0}^{-1}$, then $\psi=0$ (cf. $[25,31]$ ). The critical cases when $b$ is close to 1 or $\Theta_{0}^{-1}$ are thoroughly analyzed in $[16,14]$.

1.3. The case with a non-vanishing magnetic field. The case of a non-constant magnetic field $B_{0}$ satisfying the assumptions

$$
B_{0} \in C^{0}(\bar{\Omega}) \text { and } \inf _{x \in \bar{\Omega}} B_{0}(x)>0,
$$

is qualitatively similar to the constant magnetic field case. This situation is reviewed in $[22$, Sec. 2.2]. Surface superconductivity is studied in [15], while the transition to the normal solution is discussed in [36].

1.4. The case with a vanishing magnetic field. The results in this paper are valid for a large class of applied magnetic fields, see Assumption 1.2 below. However, one interesting situation covered by our results is the case where the applied magnetic field has a non-trivial zero set. In the presence of such an applied magnetic field, we will study the concentration of the minimizers $(\psi, \mathbf{A})_{\kappa, H}$ of (1.1) in the asymptotic limit $\kappa \rightarrow+\infty$ and $H \approx \kappa$. Unlike the results in $[15,36]$ that only investigate surface superconductivity, the situation discussed here includes bulk superconductivity as well.

The discussion in this subsection is focusing on magnetic fields that satisfy:

\section{Assumption 1.1. [On the applied magnetic field]}

(1) The function $B_{0}$ is in $C^{1}(\bar{\Omega})$.

(2) The set $\Gamma:=\left\{x \in \bar{\Omega}: B_{0}(x)=0\right\}$ is non-empty and consists of a finite disjoint union of simple smooth curves.

(3) $\Gamma \cap \partial \Omega$ is either empty or a finite set.

(4) For all $x \in \bar{\Omega},\left|B_{0}(x)\right|+\left|\nabla B_{0}(x)\right| \neq 0$. 
(5) The set $\Gamma$ is allowed to intersect $\partial \Omega$ transversely. More precisely, if $\Gamma \cap \partial \Omega \neq \emptyset$, then on this set, $\nu \times \nabla B_{0} \neq 0$, where $\nu$ is the normal vector field along $\partial \Omega$.

A much weaker assumption will be described later (cf. Assumption 1.2). Under Assumption 1.1, we may introduce the following two non-empty open sets

$$
\Omega_{+}=\left\{x \in \Omega: B_{0}(x)>0\right\} \text { and } \Omega_{-}=\left\{x \in \Omega: B_{0}(x)<0\right\} .
$$

The boundaries of $\Omega_{ \pm}$are given as follows

$$
\partial \Omega_{ \pm}=\Gamma \cup\left(\bar{\Omega}_{ \pm} \cap \partial \Omega\right) .
$$

Magnetic fields satisfying Assumption 1.1 are discussed in many contexts:

- In geometry, this appears in [32] under the appealing question: can we hear the zero locus of a magnetic field?

- In the semi-classical analysis of the spectrum of Schrödinger operators with magnetic fields satisfying Assumption 1.1 (and $\Gamma \subset \Omega$ ). These operators are extensively studied in $[13,20,24]$.

- In the study of the time-dependent Ginzburg-Landau equations $[4,5]$, applied magnetic fields as in Assumption 1.1 naturally appear in the presence of applied electric currents.

- For superconducting surfaces submitted to constant magnetic fields [11], the constant magnetic field may induce a smooth sign-changing magnetic field on the surface.

- In the transition from normal to superconducting configurations [35], one meets the problem of determining $H$ such that the ground state energy in (1.2) vanishes on a curve meeting transversally the boundary. The results in [35] are sharpened in $[9,33]$.

- The asymptotics of the ground state energy in (1.2) and the concentration of the corresponding minimizers for large values of $\kappa$ and $H$ is analyzed in [7, 8, 22, 23].

Of particular importance to us are the results of K. Attar in [7]. These results hold under Assumption 1.1, for $H=b \kappa$ with $b>0$ constant. One of the results in [7] is that the ground state energy in (1.2) satisfies, as $\kappa \rightarrow+\infty$,

$$
\mathrm{E}_{\mathrm{gs}}(\kappa, H)=\kappa^{2} \int_{\Omega} g\left(b\left|B_{0}(x)\right|\right) d x+o\left(\kappa^{2}\right) .
$$

Here the function $g(\cdot)$, which was introduced by Sandier-Serfaty in [40], is a continuous nondecreasing function defined on $[0, \infty)$ and vanishes on $[1, \infty)$ (cf. (2.5) for more details).

$\mathrm{K}$. Attar also obtained an interesting formula displaying the local distribution of the minimizing order parameter $\psi$. If $(\psi, \mathbf{A})_{\kappa, H}$ is a minimizer of the functional in (1.1) for

$$
H=b \kappa,
$$

and if $\mathcal{D}$ is an open set in $\Omega$ with a smooth boundary, then, as $\kappa \rightarrow+\infty$,

$$
\int_{\mathcal{D}}|\psi(x)|^{4} d x=-2 \int_{\mathcal{D}} g\left(b\left|B_{0}(x)\right|\right) d x+o(1) .
$$

The interest for an $L^{4}$ control of the order parameter comes back to Y. Almog (see [2] and the discussion in the book [15, Ch. 12, Sec. 12.6]).

The formula in (1.9) shows that $\psi$ is weakly localized in the neighborhood of $\Gamma, \mathcal{V}\left(\frac{1}{b}\right)$, where:

$$
\mathcal{V}(\epsilon):=\left\{x \in \Omega,\left|B_{0}(x)\right| \leq \epsilon\right\} .
$$

For taking account of the boundary effects (the surface superconductivity should play a role like in the constant magnetic field case) we also introduce in $\partial \Omega$ the subset

$$
\mathcal{V}^{\text {bnd }}(\epsilon):=\left\{x \in \partial \Omega, \Theta_{0}\left|B_{0}(x)\right| \leq \epsilon\right\} .
$$

We would like to measure the strength of the (exponential) decay of the minimizing order parameter $\psi$ in the domains

$$
\omega\left(\frac{1}{b}\right):=\Omega \backslash \mathcal{V}\left(\frac{1}{b}\right)
$$




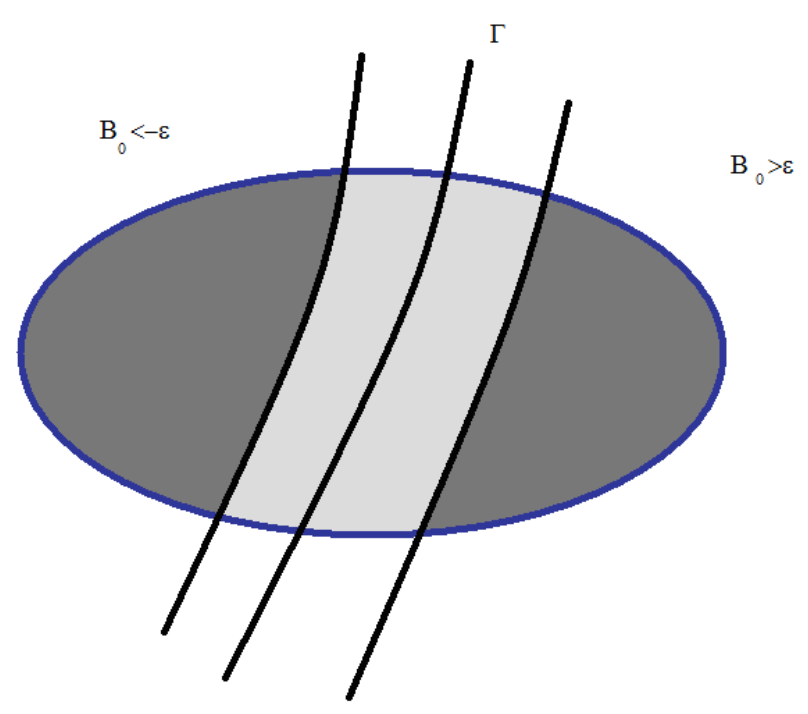

FiguRE 1. Illustration of Regime I for $H=b \kappa$ and $b=1 / \varepsilon$ : Superconductivity is destroyed in the dark regions and survived on the entire boundary.

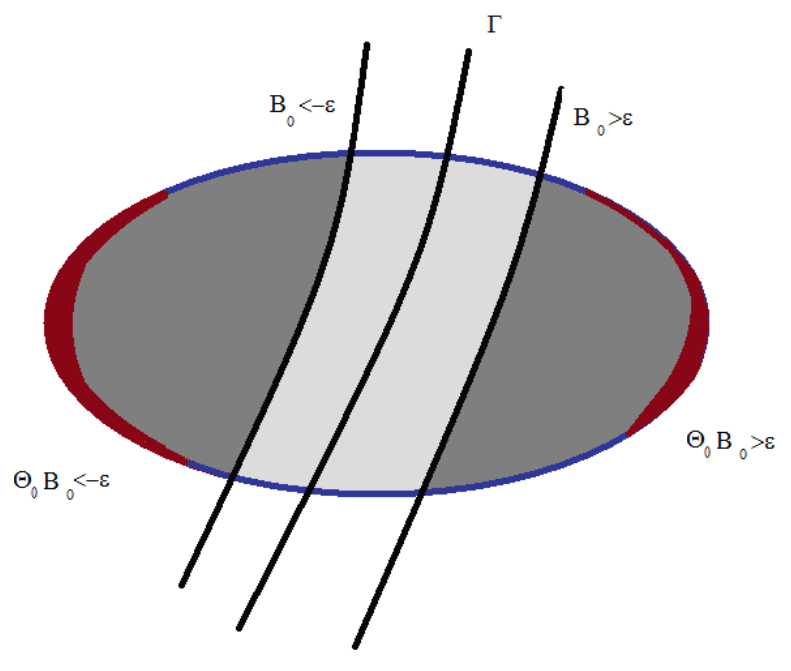

FiguRE 2. Illustration of Regime II for $H=b \kappa$ and $b=1 / \varepsilon$ : Superconductivity is also destroyed on the boundary parts $\left\{\Theta_{0}\left|B_{0}(x)\right|>\varepsilon\right\} \cap \partial \Omega$.

Note the role played by the two constants introduced in (1.4). If $\frac{1}{b} \geq \beta_{0}$, then $\mathcal{V}\left(\frac{1}{b}\right)=\Omega$. For this reason we will focus on the values of $b$ above $\beta_{0}^{-1}$. We also observe that, if $\frac{1}{b} \geq \Theta_{0} \beta_{1}$, then $\mathcal{V}^{\text {bnd }}\left(\frac{1}{b}\right)=\partial \Omega$. Hence, boundary effects are expected to appear when $b<\frac{1}{\Theta_{0} \beta_{1}}$.

Loosely speaking, we would like to prove that, for all values of $b \geq \beta_{0}^{-1}$, the density $|\psi|^{2}$ is exponentially small (in the $L^{2}$-sense) outside the set $\mathcal{V}\left(\frac{1}{b}\right) \cup \mathcal{V}^{\text {bnd }}\left(\frac{1}{b}\right)$. This will lead us to two distinct regimes:

Regime I: For $\beta_{0}^{-1}<b \leq\left(\Theta_{0} \beta_{1}\right)^{-1}, \mathcal{V}^{\text {bnd }}\left(\frac{1}{b}\right)=\partial \Omega$ and $\partial \Omega$ carries surface superconductivity everywhere. This is illustrated in Figure 1. 


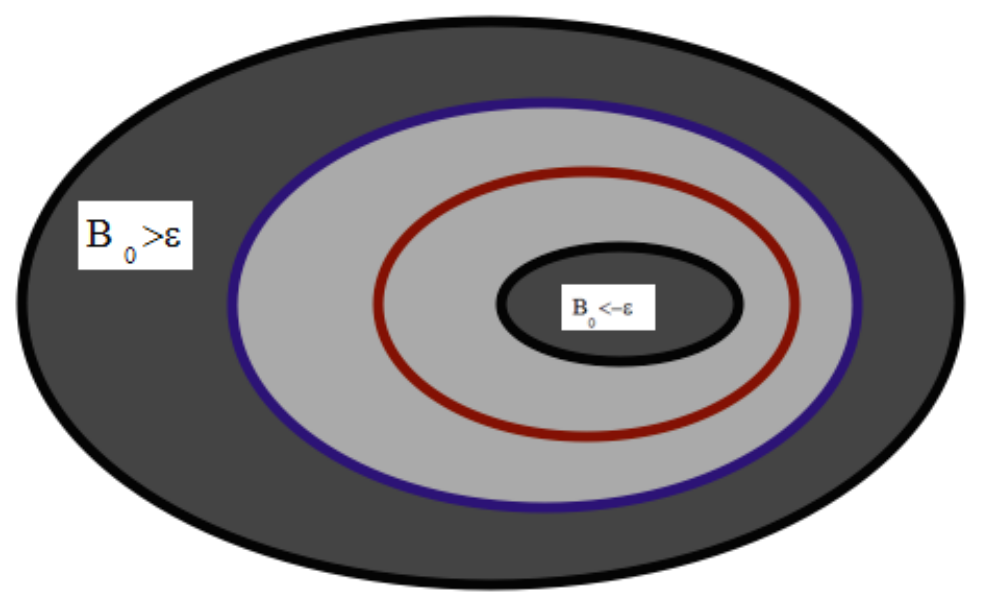

FiguRE 3. Illustration of Regime II when $\left\{B_{0}=0\right\} \cap \partial \Omega=\emptyset, H=b \kappa, b=1 / \varepsilon$ and $\varepsilon$ is small: Superconductivity is destroyed on the entire boundary and is concentrated in the set $\left\{\left|B_{0}\right|<\varepsilon\right\}$.

Regime II: For $b>\left(\Theta_{0} \beta_{1}\right)^{-1}$, we will get that $\psi$ is exponentially small outside the set $\mathcal{V}^{\text {bnd }}\left(\frac{1}{b}\right)$. Here we have two cases:

- As $b$ increases, surface superconductivity shrinks to the points of $\left\{x \in \partial \Omega, B_{0}(x)=0\right\}$, provided that this set is non-empty (cf. Figure 2).

- If $\left\{B_{0}(x)=0\right\} \cap \partial \Omega=\emptyset$, then, for sufficiently large values of $b$, no surface superconductivity is left (cf. Figure 3).

Regime II is consistent with the results of [22, Thm. 3.6] devoted to the complementary regime where $b \gg 1$ as $\kappa \rightarrow+\infty$.

The results in this paper confirm the behavior described in these two regimes and are valid under a much weaker assumption than Assumption 1.1 (cf. Assumption 1.2 below).

The transition to the normal state is studied in $[9,33,35]$. This happens, when $\kappa$ is large, for $H \sim c_{*} \kappa^{2}$ (equivalently $b \sim c_{*} \kappa$ ), where $c_{*}>0$ is a constant explicitly defined by the domain $\Omega$ and the function $B_{0}$.

\subsection{Main results.}

In this paper, we will first work under the following assumption:

\section{Assumption 1.2.}

- The function $B_{0}$ is in $C^{0, \alpha}(\bar{\Omega})$ for some $\alpha \in(0,1)$;

- The constants $\beta_{0}$ and $\beta_{1}$ in (1.4) satisfy $\beta_{1} \geq \beta_{0}>0$.

Note that this assumption is much weaker than Assumption 1.1. With the previous notation our main theorem is:

\section{Theorem 1.3. [Exponential decay outside the superconductivity region]}

Suppose that Assumption 1.2 holds, that $b>\beta_{0}^{-1}$ and let $O$ be an open set such that $\bar{O} \subset \omega\left(\frac{1}{b}\right)$, where $\omega\left(\frac{1}{b}\right)$ is the domain introduced in $(1.12)$

There exist $\kappa_{0}>0, C>0$ and $\alpha_{0}>0$ such that, if $\kappa \geq \kappa_{0}$ and $(\psi, \mathbf{A})_{\kappa, H}$ is a solution of (1.3) for $H=b \kappa$, then the following inequality holds

$$
\|\psi\|_{H^{1}(O)} \leq C e^{-\alpha_{0} \kappa} .
$$

Furthermore, if $b>\left(\Theta_{0} \beta_{1}\right)^{-1}$, then the estimate in (1.13) holds when the open set $O$ satisfies

$$
\bar{O} \subset\left\{x \in \partial \Omega, \Theta_{0}\left|B_{0}(x)\right|>\frac{1}{b}\right\} \cup \omega\left(\frac{1}{b}\right) .
$$


The proof of Theorem 1.3 follows from the stronger conclusion of Theorem 3.1, establishing Agmon like estimates.

\section{Remark 1.4. [Sign-changing magnetic fields]}

In addition to Assumption 1.2, suppose that $\Omega_{+}$and $\Omega_{-}$are non-empty. The constant $\beta_{0}$ in (1.4) can be expressed as follows

$$
\beta_{0}=\max \left(\beta_{0}^{+}, \beta_{0}^{-}\right) \quad \text { where } \beta_{0}^{ \pm}=\sup _{x \in \overline{\Omega_{ \pm}}}\left|B_{0}(x)\right| .
$$

We will discuss the conclusion of Theorem 1.3 when $\beta_{0}^{+}<\beta_{0}^{-}$. We have:

- If $\left(\beta_{0}\right)^{-1}<b<\left(\beta_{0}^{+}\right)^{-1}$, then $\omega\left(\frac{1}{b}\right) \cap \Omega_{+}=\emptyset$. Consequently, the exponential decay occurs in $\omega\left(\frac{1}{b}\right) \cap \Omega_{-}$.

- If $\left(\beta_{0}^{+}\right)^{-1} \leq b$, then the exponential decay occurs in both $\omega\left(\frac{1}{b}\right) \cap \Omega_{+}$and $\omega\left(\frac{1}{b}\right) \cap \Omega_{-}$.

The situation when $\beta_{0}^{-}<\beta_{0}^{+}$can be discussed similarly. Next, we suppose that the two sets

$$
(\partial \Omega)_{+}:=\left\{x \in \partial \Omega, B_{0}(x)>0\right\} \text { and }(\partial \Omega)_{-}:=\left\{x \in \partial \Omega, B_{0}(x)<0\right\}
$$

are non-empty, and we express the constant $\beta_{1}$ in (1.4) as follows

$$
\beta_{1}=\max \left(\beta_{1}^{+}, \beta_{1}^{-}\right) \quad \text { where } \beta_{1}^{ \pm}=\sup _{x \in \overline{(\partial \Omega)_{ \pm}}}\left|B_{0}(x)\right| .
$$

According to Theorem 1.3, when $\beta_{1}^{+}<\beta_{1}^{-}$and $\left(\beta_{1}\right)^{-1}<b<\left(\beta_{1}^{+}\right)^{-1}$, then the exponential decay occurs on $\left\{x \in \partial \Omega, \Theta_{0} b\left|B_{0}(x)\right|>1\right\} \cap(\partial \Omega)_{-}$, since $\left\{x \in \partial \Omega, \Theta_{0} b\left|B_{0}(x)\right|>1\right\} \cap(\partial \Omega)_{+}=\emptyset$.

Our next result discusses the optimality of Theorem 1.3. This theorem determines a part of the boundary where the order parameter (the first component $\psi$ of the minimizer) is exponentially small. Outside this part of the boundary, we will prove that the $L^{4}$ norm of the order parameter is not exponentially small. In physical terms, superconductivity is present there.

The statement of Theorem 1.5 involves the following notation:

- For all $t>0, \widetilde{\Omega}(t)=\left\{x \in \mathbb{R}^{2}: \operatorname{dist}(x, \partial \Omega)<t\right\}$.

- By smoothness of $\partial \Omega$, there exists a geometric constant $t_{0}$ such that, for all $x \in \widetilde{\Omega}\left(t_{0}\right)$, we may assign a unique point $p(x) \in \partial \Omega$ such that $\operatorname{dist}(p(x), x)=\operatorname{dist}(x, \partial \Omega)$.

- If $b>0$, we define the open subset in $\mathbb{R}^{2}$

$$
\widetilde{\Omega}\left(t_{0}, b\right)=\left\{x \in \widetilde{\Omega}\left(t_{0}\right): 1<b\left|B_{0}(p(x))\right|<\Theta_{0}^{-1}\right\} .
$$

- $E_{\text {surf }}:\left[1, \Theta_{0}^{-1}\right) \rightarrow(-\infty, 0)$ is the surface energy function which will be defined in (4.5) later. This function is continuous and non-decreasing.

- If $\widetilde{\Omega}\left(t_{0}, b\right) \neq \emptyset$, we define the following distribution in $\mathcal{D}^{\prime}\left(\widetilde{\Omega}\left(t_{0}, b\right)\right)$ :

$$
C_{c}^{\infty}\left(\widetilde{\Omega}\left(t_{0}, b\right)\right) \ni \varphi \mapsto \mathcal{T}_{b}(\varphi)=-2 \int_{\widetilde{\Omega}\left(t_{0}, b\right) \cap \partial \Omega} \sqrt{\frac{1}{b\left|B_{0}(x)\right|}} E_{\text {surf }}\left(b\left|B_{0}(x)\right|\right) \varphi(x) d s(x),
$$

where $d s$ is the surface measure on $\partial \Omega$.

- If $D \subset \bar{\Omega}$, we introduce the local Ginzburg-Landau energy in $D$ as follows

$$
\mathcal{E}(\psi, \mathbf{A} ; D)=\int_{D}\left(|(\nabla-i \kappa H \mathbf{A}) \psi|^{2}-\kappa^{2}|\psi|^{2}+\frac{\kappa^{2}}{2}|\psi(x)|^{4}\right) d x .
$$

- $\mathbf{1}_{\Omega}$ denotes the characteristic function of the set $\Omega$.

\section{Theorem 1.5. [Existence of surface superconductivity]}

Suppose that Assumption 1.2 holds, that $b>\beta_{0}^{-1}$ and that $\widetilde{\Omega}\left(t_{0}, b\right) \neq \emptyset$, where $\beta_{0}$ is the constant introduced in (1.4). If $(\psi, \mathbf{A})_{\kappa, H}$ is a minimizer of the functional in (1.1) for $H=b \kappa$, then as $\kappa \rightarrow \infty$, we have the following weak convergence

$$
\kappa \mathbf{1}_{\Omega}\left|\psi_{\kappa, H}\right|^{4} \rightarrow \mathcal{T}_{b} \quad \text { in } \mathcal{D}^{\prime}\left(\widetilde{\Omega}\left(t_{0}, b\right)\right) .
$$


Remark 1.6. Theorem 1.5 demonstrates the existence of surface superconductivity. We can interpret the assumption in Theorem 4.8 in two different ways.

- If $H=b \kappa, b>0$ is fixed and $x_{0} \in \partial \Omega$, then to find superconductivity near $x_{0}$, this point should satisfy $1<b\left|B_{0}\left(x_{0}\right)\right|<\Theta_{0}^{-1}$.

- If $x_{0} \in \partial \Omega$ is fixed and $\left|B_{0}\left(x_{0}\right)\right|$ is small, then to find superconductivity near $x_{0}$, the intensity of the applied magnetic field should be increased in such a manner that $H=b \kappa$ and $1<b\left|B_{0}\left(x_{0}\right)\right|<\Theta_{0}^{-1}$.

Our last result confirms that the region $\left\{B_{0}(x)<\frac{\kappa}{H}\right\}$ carries superconductivity everywhere. To state it, we will use the following notation:

- If $p, q \in \partial \Omega$, $\operatorname{dist}_{\partial \Omega}(p, q)$ denotes the (arc-length) distance in $\partial \Omega$ between $p$ and $q$.

- For $x_{0} \in \mathbb{R}^{2}$ and $r>0$, we denote by $Q_{r}\left(x_{0}\right)=x_{0}+(-r / 2, r / 2)^{2}$ the interior of the square of center $x_{0}$ and side $r$. When $x_{0}=0$, we write $Q_{r}=Q_{r}(0)$.

- For $(x, \ell) \in \bar{\Omega} \times\left(0, t_{0} / 2\right)$, we will use the following notation:

$$
\mathcal{W}\left(x_{0}, \ell\right)=\left\{\begin{array}{cl}
\left\{x \in \bar{\Omega}: \operatorname{dist}_{\partial \Omega}\left(p(x), x_{0}\right)<\ell \text { and } \operatorname{dist}(x, \partial \Omega)<2 \ell\right\} & \text { if } x_{0} \in \partial \Omega, \\
Q_{2 \ell}\left(x_{0}\right) & \text { if } x_{0} \in \Omega .
\end{array}\right.
$$

\section{Theorem 1.7. [The superconductivity region]}

Suppose that Assumption 1.2 holds for some $\alpha \in(0,1), b>0$ and $\frac{2}{2+\alpha}<\rho<1$ be two constants. Let $x_{0} \in \bar{\Omega}$ such that $\left|B_{0}\left(x_{0}\right)\right|<\frac{1}{b}$.

There exist $\kappa_{0}>0$, a function $\mathrm{r}:\left[\kappa_{0},+\infty\right) \rightarrow \mathbb{R}_{+}$such that $\lim _{\kappa \rightarrow+\infty} \mathrm{r}(\kappa)=0$ and, for all $\kappa \geq \kappa_{0}$ and for all critical point $(\psi, \mathbf{A})_{\kappa, H}$ of the functional in (1.1) with $H=b \kappa$, the following two inequalities hold,

$$
\left.\left|\frac{1}{\left|\mathcal{W}\left(x_{0}, \kappa^{-\rho}\right)\right|} \int_{\mathcal{W}_{x_{0}}\left(\kappa^{-\rho}\right)}\right| \psi(x)\right|^{4} d x+2 g\left(b\left|B_{0}\left(x_{0}\right)\right|\right) \mid \leq \mathrm{r}(\kappa)
$$

and

$$
\left|\mathcal{E}\left(\psi, \mathbf{A} ; \mathcal{W}\left(x_{0}, \kappa^{-\rho}\right)\right)-\kappa^{2} g\left(b\left|B_{0}\left(x_{0}\right)\right|\right)\right| \leq \kappa^{2} \mathrm{r}(\kappa) .
$$

Here $g(\cdot)$ is the continuous function appearing in (1.8) and (1.9) (see Subsection 2.1 for its definition and properties).

The result in Theorem 1.7 is a variant of the formula in (1.9) valid for applied magnetic fields which are only Hölder continuous, thereby generalizing the results by Attar [7] and SandierSerfaty [40]. This will be clarified further in Remark 1.9.

Remark 1.8. Let us choose fixed constants $\gamma$ and $\rho$ such that $\frac{2}{2+\alpha}<\rho<1$ and $0<\gamma<1-\rho$. Our proof of Theorem 1.7 yields that the constant $\kappa_{0}$ and the function $r(\kappa)$ in Theorem 1.7 can be selected independently of the point $x_{0}$ provided that

- $\kappa^{-2 \gamma} \leq b\left|B_{0}\left(x_{0}\right)\right|<1$;

- $x_{0} \in \partial \Omega$ or $\operatorname{dist}\left(x_{0}, \partial \Omega\right) \geq 4 \kappa^{-\rho}$.

The condition $\operatorname{dist}\left(x_{0}, \partial \Omega\right) \geq 4 \kappa^{-\rho}$ ensures that $Q_{x_{0}}\left(2 \kappa^{-\rho}\right) \subset \bar{\Omega}$, which is needed in the proof of Theorem 1.7.

Remark 1.9. Let $\gamma \in\left(0, \frac{\alpha}{2+\alpha}\right)$. If we assume furthermore the following geometric condition

$$
\left|\left\{x \in \bar{\Omega},\left|B_{0}(x)\right| \leq \kappa^{-2 \gamma}\right\}\right|=o(1) \quad(\kappa \rightarrow \infty),
$$

then Theorem 1.7 implies the weak convergence

$$
\left|\psi_{\kappa, H}(\cdot)\right|^{4} \rightarrow-2 g\left(b\left|B_{0}(\cdot)\right|\right) \quad \text { in } \mathcal{D}^{\prime}(\Omega) .
$$

In (1.19), we used the following notation. If $E \subset \mathbb{R}^{2},|E|$ denotes the Lebesgue (area) measure of $E$. Note that the condition in (1.19) holds under Assumption 1.1 considered in [7]. 
The rest of the paper is organized as follows. In Section 2, we collect various results that will be used throughout the paper. Section 3 is devoted to the proof of Theorem 1.3. In Section 4, we present the proof of Theorem 1.5. Finally, we prove Theorem 1.7 in Section 5.

In the proofs, we avoid the use of the a priori elliptic $L^{\infty}$-estimates, whose derivation is quite complicated (cf. [15, Ch. 11]), thereby providing new proofs for the results in [34, 40]. To our knowledge, these $L^{\infty}$-estimates have not been established when the magnetic field $B_{0}$ is only Hölder continuous.

\section{Preliminaries}

2.1. The bulk energy function. The energy function $g(\cdot)$, hereafter called the bulk energy, has been constructed in [40]. We will recall its construction here. It plays a central role in the study of 'bulk' superconductivity, both for two and three dimensional problems (cf. [19, 17]). Furthermore, it is related to the periodic solutions of (1.3) and the Abrikosov energy (cf. $[1,16])$.

For $b \in(0,+\infty), r>0$, and $Q_{r}=(-r / 2, r / 2) \times(-r / 2, r / 2)$, we define the functional,

$$
F_{b, Q_{r}}(u)=\int_{Q_{r}}\left(b\left|\left(\nabla-i \mathbf{A}_{0}\right) u\right|^{2}-|u|^{2}+\frac{1}{2}|u|^{4}\right) d x, \quad \text { for } u \in H^{1}\left(Q_{r}\right) .
$$

Here, $\mathbf{A}_{0}$ is the magnetic potential,

$$
\mathbf{A}_{0}(x)=\frac{1}{2}\left(-x_{2}, x_{1}\right), \quad \text { for } x=\left(x_{1}, x_{2}\right) \in \mathbb{R}^{2} .
$$

We define the Dirichlet and Neumann ground state energies by

$$
\begin{aligned}
& e_{D}(b, r)=\inf \left\{F_{b, Q_{r}}(u): u \in H_{0}^{1}\left(Q_{r}\right)\right\}, \\
& e_{N}(b, r)=\inf \left\{F_{b, Q_{r}}(u): u \in H^{1}\left(Q_{r}\right)\right\} .
\end{aligned}
$$

We can define $g(\cdot)$ as follows $($ cf. $[7,17,40])$

$$
\forall b>0, \quad g(b)=\lim _{r \rightarrow \infty} \frac{e_{D}(b, r)}{\left|Q_{r}\right|}=\lim _{r \rightarrow \infty} \frac{e_{N}(b, r)}{\left|Q_{r}\right|},
$$

where $\left|Q_{r}\right|=r^{2}$ denotes the area of $Q_{r}$.

Furthermore, there exists a universal constant $C>0$ such that

$$
\forall b>0, \quad \forall r>1, \quad g(b) \leq \frac{e_{D}(b, r)}{\left|Q_{r}\right|} \leq \frac{e_{N}(b, r)}{\left|Q_{r}\right|}+\frac{C}{r} \leq g(b)+\frac{2 C}{r} .
$$

One can show that the function $g(\cdot)$ is a non decreasing continuous function such that

$$
g(0)=-\frac{1}{2}, \quad g(b)<0 \text { when } b<1, \text { and } \quad g(b)=0 \text { when } b \geq 1 .
$$

2.2. The magnetic Laplacian. We need two results about the magnetic Laplacian. The first result concerns the Dirichlet magnetic Laplace operator in a bounded set $\Omega$ with a strong constant magnetic field $B$, that is

with the Dirichlet condition

$$
-\left(\nabla-i B \mathbf{A}_{0}\right)^{2} \quad \text { in } L^{2}(\Omega)
$$

$$
u=0 \text { on } \partial \Omega \text {. }
$$

Here $\mathbf{A}_{0}$ is the vector field introduced in (2.2), with curl $\mathbf{A}_{0}=1$. It is based on the elementary spectral inequality:

Lemma 2.1. For all $B \in \mathbb{R}$ and $\phi \in H_{0}^{1}(\Omega)$, it holds

$$
\int_{\Omega}\left|\left(\nabla-i B \mathbf{A}_{0}\right) \phi\right|^{2} d x \geq|B| \int_{\Omega}|\phi(x)|^{2} d x .
$$


The second result concerns the Neumann magnetic Laplace operator in a bounded set $\Omega$ with a strong constant magnetic field $B$, that is

$$
-\left(\nabla-i B \mathbf{A}_{0}\right)^{2} \quad \text { in } L^{2}(\Omega),
$$

with the (magnetic) Neumann condition

$$
\nu \cdot\left(\nabla-i B \mathbf{A}_{0}\right) u=0 \text { on } \partial \Omega .
$$

Here $\nu$ is the unit inward normal vector on $\partial \Omega$. The asymptotic behavior of the groundstate energy as $|B| \rightarrow \infty$ is well known (cf. [21,30] and [15, Prop. 8.2.2]):

Lemma 2.2. There exist $\hat{\beta}_{0}>0$ and $C>0$ such that, if $|B| \geq \hat{\beta}_{0}$ and $\phi \in H^{1}(\Omega)$,

$$
\int_{\Omega}\left|\left(\nabla-i B \mathbf{A}_{0}\right) \phi\right|^{2} d x \geq\left(\Theta_{0}|B|-C|B|^{3 / 4}\right) \int_{\Omega}|\phi|^{2} d x .
$$

2.3. Universal bound on the order parameter. If $(\psi, \mathbf{A})$ is a solution of (1.3), then $\psi$ satisfies in $\Omega$ (cf. [15, Prop. 10.3.1])

$$
|\psi(x)| \leq 1 .
$$

2.4. The magnetic energy. Let us introduce the space of vector fields

$$
H_{\text {div }}^{1}(\Omega)=\left\{\mathbf{A} \in H^{1}\left(\Omega ; \mathbb{R}^{2}\right): \operatorname{div} \mathbf{A}=0 \text { in } \Omega \text { and } \nu \cdot \mathbf{A}=0 \text { on } \partial \Omega\right\} .
$$

The functional in (1.1) is invariant under the gauge transformations $(\psi, \mathbf{A}) \mapsto\left(e^{i \phi} \psi, \mathbf{A}+\nabla \phi\right)$. Consequently, if $(\psi, \mathbf{A})$ solves (1.3), we may apply a gauge transformation such that the new configuration $\left(\widetilde{\psi}=e^{i \phi} \psi, \widetilde{A}=A+\nabla \phi\right)$ is a solution of (1.3) and furthermore $\widetilde{\mathbf{A}} \in H_{\text {div }}^{1}(\Omega)$. Having this in hand, we always assume that every critical/minimizing configuration $(\psi, \mathbf{A})$ satisfies $\mathbf{A} \in H_{\text {div }}^{1}(\Omega)$ which simply amounts to a gauge transformation.

For given $B_{0} \in L^{2}(\Omega)$, there exists a unique vector field satisfying

$$
\mathbf{F} \in H_{\text {div }}^{1}(\Omega) \text { and } \operatorname{curl} \mathbf{F}=B_{0} .
$$

Actually, $\mathbf{F}=\nabla^{\perp} f$ where $f \in H^{2}(\Omega) \cap H_{0}^{1}(\Omega)$ is the unique solution of $-\Delta f=B_{0}$.

Remark 2.3. By the elliptic Schauder Hölder estimates (see for example Appendix E.3 in [15]), if in addition $B_{0} \in C^{0, \alpha}(\bar{\Omega})$ for some $\alpha>0$, then the vector field $\mathbf{F}$ is smooth of class $C^{1, \alpha}(\bar{\Omega})$.

We recall the following result from [7]:

Proposition 2.4. Let $\gamma \in(0,1)$ and $0<c_{1}<c_{2}$ be fixed constants. Suppose that $B_{0} \in L^{2}(\Omega)$. There exist $\kappa_{0}>0$ and $C>0$ such that, if $\kappa \geq \kappa_{0}, c_{1} \kappa \leq H \leq c_{2} \kappa$ and if $(\psi, \mathbf{A})_{\kappa, H} \in$ $H^{1}(\Omega) \times H_{\mathrm{div}}^{1}(\Omega)$ is a minimizer of $(1.2)$, then

$$
\|\mathbf{A}-\mathbf{F}\|_{C^{0, \gamma}(\bar{\Omega})} \leq \frac{C}{\kappa} .
$$

The proof of Proposition 2.4 given in [7] is made under the assumption $B_{0} \in C^{\infty}(\bar{\Omega})$, but it still holds under the weaker assumption $B_{0} \in L^{2}(\Omega)$.

The next result gives the existence of a useful gauge transformation that allows us to approximate the vector field $\mathbf{F}$ by a vector field generating a constant magnetic field. It is similar to the result in [7, Lem. A.3], but the difference here is that we only assume $\mathbf{F} \in C^{1, \alpha}(\bar{\Omega})$ instead of $C^{2}$.

Lemma 2.5. Let $\alpha \in(0,1), r_{0}>0$ and $B_{0} \in C^{0, \alpha}(\bar{\Omega})$. There exists $C>0$ and for any $a \in \bar{\Omega} a$ function $\varphi_{a} \in C^{2, \alpha}\left(\mathbb{R}^{2}\right)$ such that, if $r \in\left(0, r_{0}\right]$ and $B(a, r) \cap \Omega \neq \emptyset$, then

$$
\forall x \in \overline{B(a, r) \cap \Omega}, \quad\left|\mathbf{F}(x)-B_{0}(a) \mathbf{A}_{0}(x-a)-\nabla \varphi_{a}(x)\right| \leq C r^{1+\alpha} .
$$

Here $\mathbf{F}$ is the vector field satisfying (2.10). 
Proof of Lemma 2.5. Since the boundary of $\Omega$ is smooth and $\mathbf{F} \in C^{1, \alpha}\left(\bar{\Omega} ; \mathbb{R}^{2}\right)$, the vector field $\mathbf{F}$ admits an extension $\widehat{\mathbf{F}}$ in $C^{1, \alpha}\left(\mathbb{R}^{2} ; \mathbb{R}^{2}\right)$. We get in this way an extension $\widehat{B}_{0}=\operatorname{curl} \widehat{\mathbf{F}}$ of $B_{0}$ in $C^{0, \alpha}\left(\mathbb{R}^{2}\right)$. We now define in $\mathbb{R}^{2}$, the two vector fields

$$
\widetilde{\mathbf{F}}(y)=\widehat{\mathbf{F}}(a+y), \quad \widetilde{\mathbf{A}}(y)=\left(\int_{0}^{1} s \widehat{B}_{0}(a+s y) d s\right)\left(-y_{2}, y_{1}\right) .
$$

Clearly, curl $\widetilde{\mathbf{F}}=\operatorname{curl} \widetilde{\mathbf{A}}=\widehat{B}_{0}(a+y)$. Consequently, by integrating the closed 1-form associated with $\widetilde{F}-\widetilde{\mathbf{A}}$, there exists a function $\widetilde{\varphi} \in C^{2, \alpha}\left(\mathbb{R}^{2}\right)$ such that

$$
\widetilde{\mathbf{F}}-\nabla \widetilde{\varphi}=\widetilde{\mathbf{A}}, \widetilde{\phi}(0)=0 .
$$

Since $\widehat{B}_{0} \in C^{0, \alpha}\left(\mathbb{R}^{2}\right), \widetilde{\mathbf{A}}(y)=B_{0}(a)\left(-y_{2}, y_{1}\right)+\mathcal{O}\left(r^{1+\alpha}\right)$ in $\overline{B(0, r)}$. We then define the function $\varphi_{a}$ by $\varphi_{a}(x)=\widetilde{\varphi}(x-a)+B_{0}(a)\left(a_{2} x_{1}-a_{1} x_{2}\right)$. This implies

$$
\forall x \in \overline{B(a, r)}, \quad\left|\widehat{\mathbf{F}}(x)-B_{0}(a) \mathbf{A}_{0}(x-a)-\nabla \varphi_{a}(x)\right| \leq C r^{1+\alpha},
$$

and the lemma by restriction to $\bar{\Omega}$.

2.5. Lower bound of the kinetic energy term. The main result in this subsection is:

Proposition 2.6. Let $0<c_{1}<c_{2}$ be fixed constants. Suppose that $\alpha \in(0,1]$ and $B_{0} \in C^{0, \alpha}(\bar{\Omega})$. There exist $\kappa_{0}>0$ and $C>0$ such that the following is true, with

$$
\sigma(\alpha)=\frac{2 \alpha}{3+\alpha} .
$$

(1) For

- $\kappa \geq \kappa_{0}, c_{1} \kappa \leq H \leq c_{2} \kappa$;

- $(\psi, \mathbf{A})_{\kappa, H}$ a solution of $(1.3)$;

- $\phi \in H^{1}(\Omega)$ satisfies $\operatorname{supp} \phi \subset\left\{x \in \bar{\Omega},\left|B_{0}(x)\right|>0\right\}$, we have

$$
\int_{\Omega}|(\nabla-i \kappa H \mathbf{A}) \phi(x)|^{2} d x \geq \Theta_{0} \kappa H \int_{\Omega}\left(\left|B_{0}(x)\right|-C \kappa^{-\sigma(\alpha)}\right)|\phi(x)|^{2} d x .
$$

(2) If in addition $\phi=0$ on $\partial \Omega$, then

$$
\int_{\Omega}|(\nabla-i \kappa H \mathbf{A}) \phi(x)|^{2} d x \geq \kappa H \int_{\Omega}\left(\left|B_{0}(x)\right|-C \kappa^{-\sigma(\alpha)}\right)|\phi(x)|^{2} d x .
$$

The estimates in Items (1) and (2) in this proposition are known when the vector field $\mathbf{A}$ is $C^{2}$, independent of $(\kappa, H)$, curl $\mathbf{A} \neq 0$ and $B_{0}$ is replaced by curl $\mathbf{A}$ (cf. Lemma 2.2 and [20]).

For $\alpha=1$ (i.e. $B_{0}$ is Lipschitz) the errors in Proposition 2.6 and Lemma 2.2 are of the same order.

Proof of Proposition 2.6. Let us choose an arbitrary $\phi \in H^{1}(\Omega)$. All constants below are independent of $\phi$. For the sake of simplicity, we will work under the additional assumption that $\operatorname{supp} \phi \subset\left\{B_{0}>0\right\}$.

Step 1. Decomposition of the energy via a partition of unity.

For $\ell>0$ we consider the partition of unity in $\mathbb{R}^{2}$

$$
\sum_{j} \chi_{j}^{2}=1, \quad \sum_{j}\left|\nabla \chi_{j}\right|^{2} \leq C \ell^{-2} \quad \text { in } \mathbb{R}^{2}, \quad \text { and } \quad \operatorname{supp} \chi_{j} \subset B\left(a_{j}^{\ell}, \ell\right) .
$$

Here the construction is first done for $\ell=1$ and then for general $\ell>0$ by dilation. Hence the constant $C$ is independent of $\ell$. Although the points $\left(a_{j}^{\ell}\right)$ depend on $\ell$, we omit below the reference to $\ell$ and write $a_{j}$ for $a_{j}^{\ell}$. 
In what follows, we will use this partition of unity with

$$
\ell=\kappa^{-\rho}, \quad 0<\rho<1 \text { and } \kappa \text { large enough. }
$$

Using this partition of unity, we may estimate from below the kinetic energy term as follows

$$
\int_{\Omega}|(\nabla-i \kappa H \mathbf{A}) \phi|^{2} d x \geq \sum_{j}\left(\int_{\Omega}\left|(\nabla-i \kappa H \mathbf{A})\left(\chi_{j} \phi\right)\right|^{2} d x-C \ell^{-2} \int_{\Omega}\left|\chi_{j} \phi\right|^{2} d x\right) .
$$

Let $\alpha_{j}(x)=\left(x-a_{j}\right) \cdot\left(\mathbf{A}\left(a_{j}\right)-\mathbf{F}\left(a_{j}\right)\right)$, where $\mathbf{F}$ is the vector field in (2.10). Note the useful decomposition

$$
\mathbf{A}(x)-\nabla \alpha_{j}=\mathbf{F}(x)+(\mathbf{A}(x)-\mathbf{F}(x))-\left(\mathbf{A}\left(a_{j}\right)-\mathbf{F}\left(a_{j}\right)\right) .
$$

By Proposition 2.4, we have in $B\left(a_{j}, \ell\right) \cap \Omega$,

$$
\begin{aligned}
\left|(\nabla-i \kappa H \mathbf{A})\left(\chi_{j} \phi\right)\right|^{2} & =\left|\left(\nabla-i \kappa H\left(\mathbf{A}-\nabla \alpha_{j}\right)\right)\left(e^{-i \kappa H \alpha_{j}} \chi_{j} \phi\right)\right|^{2} \\
& \geq\left(1-\ell^{\delta}\right)\left|(\nabla-i \kappa H \mathbf{F}) e^{-i \kappa H \alpha_{j}} \chi_{j} \phi\right|^{2}-\ell^{-\delta} \kappa^{2} H^{2} \ell^{2 \gamma}\|\mathbf{A}-\mathbf{F}\|_{C^{0, \gamma}(\bar{\Omega})}^{2}\left|\chi_{j} \phi\right|^{2} \\
& \geq\left(1-\ell^{\delta}\right)\left|(\nabla-i \kappa H \mathbf{F})\left(e^{-i \kappa H \alpha_{j}} \chi_{j} \phi\right)\right|^{2}-C H^{2} \ell^{(2 \gamma-\delta)}\left|\chi_{j} \phi\right|^{2} .
\end{aligned}
$$

Here $\delta>0$ and $\gamma \in(0,1)$ are two parameters to be chosen later.

By Lemma 2.5, we may define a smooth function $\varphi_{j}$ in $B\left(a_{j}, \ell\right) \cap \Omega$ such that,

$$
\left|\mathbf{F}(x)-\nabla \varphi_{j}(x)-\right| B_{0}\left(a_{j}\right)\left|\mathbf{A}_{0}\left(x-a_{j}\right)\right| \leq C \ell^{1+\alpha},
$$

where $C>0$ is independent of $j$.

Consequently, there exists $C>0$ such that, for all $j$,

$$
\begin{array}{r}
\left|(\nabla-i \kappa H \mathbf{F})\left(e^{-i \kappa H \alpha_{j}} \chi_{j} \phi\right)\right|^{2} \geq\left(1-\ell^{\delta}\right)\left|\left(\nabla-i \kappa H\left|B_{0}\left(a_{j}\right)\right| \mathbf{A}_{0}\left(x-a_{j}\right)\right) e^{-i \kappa H \varphi_{j}} e^{-i \kappa H \alpha_{j}} \chi_{j} \phi\right|^{2} \\
-C \kappa^{2} H^{2} \ell^{2+2 \alpha-\delta}\left|\chi_{j} \phi\right|^{2} .
\end{array}
$$

Step 2. The case $\operatorname{supp} \phi \subset\left\{x \in \bar{\Omega}, B_{0}(x)>0\right\}$ and $\phi=0$ on $\partial \Omega$.

The assumption on the support of $\phi$ yields that $\chi_{j} \phi \in H_{0}^{1}(\Omega)$. Collecting (2.13), (2.14) and the spectral inequality in Lemma 2.1, we get the existence of $C>0$ such that for all $j$

$$
\begin{gathered}
\int_{\Omega}\left|(\nabla-i \kappa H \mathbf{A})\left(\chi_{j} \phi\right)\right|^{2} d x \geq\left(1-2 \ell^{\delta}\right) \kappa H \int_{\Omega}\left|B_{0}\left(a_{j}\right)\right|\left|\chi_{j} \phi\right|^{2} d x \\
-C H^{2}\left(\ell^{2 \gamma-\delta}+\kappa^{2} \ell^{2+2 \alpha-\delta}\right) \int_{\Omega}\left|\chi_{j} \phi\right|^{2} d x .
\end{gathered}
$$

Since $B_{0}$ is in $C^{0, \alpha}(\bar{\Omega})$, we have $B_{0}(x)=B_{0}\left(a_{j}\right)+\mathcal{O}\left(\ell^{\alpha}\right)$ in $B\left(a_{j}, \ell\right)$. Thus

$$
\begin{aligned}
\int_{\Omega}\left|(\nabla-i \kappa H \mathbf{A})\left(\chi_{j} \phi\right)\right|^{2} d x \geq \kappa H \int_{\Omega} \mid & \left.B_{0}(x)|| \chi_{j} \phi(x)\right|^{2} d x \\
& -C H^{2}\left(\ell^{\alpha}+\ell^{\delta}+\ell^{2 \gamma-\delta}+\kappa^{2} \ell^{2+2 \alpha-\delta}\right) \int_{\Omega}\left|\chi_{j} \phi(x)\right|^{2} d x .
\end{aligned}
$$

After summation and using that $\sum_{j} \chi_{j}^{2}=1$, we get

$$
\begin{aligned}
\int_{\Omega} \mid(\nabla & -i \kappa H \mathbf{A})\left.\phi\right|^{2} d x \\
& \geq \kappa H\left(\int_{\Omega}\left|B_{0}(x)\right||\phi(x)|^{2} d x-C\left(\ell^{\alpha}+\ell^{\delta}+\ell^{2 \gamma-\delta}+\kappa^{2} \ell^{2+2 \alpha-\delta}+\kappa^{-2} \ell^{-2}\right) \int_{\Omega}|\phi|^{2} d x\right) .
\end{aligned}
$$

Hence the goal is to choose, when $\kappa \rightarrow+\infty$ and with $\ell=\kappa^{-\rho}$, the parameters $\rho, \delta, \gamma$ and $\alpha$ in order to minimize the sum

$$
\Sigma_{0}(\kappa, \ell):=\ell^{\alpha}+\ell^{\delta}+\ell^{2 \gamma-\delta}+\kappa^{2} \ell^{2+2 \alpha-\delta}+\kappa^{-2} \ell^{-2} .
$$


If we take $\delta=\gamma$, which corresponds to give the same order for the second and the third terms in $\Sigma_{0}$, we obtain with $\ell=\kappa^{-\rho}$

$$
\int_{\Omega}|(\nabla-i \kappa H \mathbf{A}) \phi|^{2} d x \geq \kappa H \int_{\Omega}\left(\left|B_{0}(x)\right|-C\left(\kappa^{-\rho \alpha}+\kappa^{-\rho \gamma}+\kappa^{2-(2+2 \alpha-\gamma) \rho}+\kappa^{2 \rho-2}\right)|\phi(x)|^{2} d x .\right.
$$

In the remainder, to minimize the error for the two last terms, we select $\rho$ such that

$$
2-(2+2 \alpha-\gamma) \rho=2 \rho-2,
$$

i.e.

$$
\rho=4 /(4+2 \alpha-\gamma)
$$

Getting the condition $0<\rho<1$ satisfied leads to the condition $\alpha>\gamma / 2$. We select $\gamma=\frac{2}{3} \alpha$. This choice is optimal since

$$
\sigma(\alpha):=\max _{0<\gamma<2 \alpha} \sigma_{0}(\alpha, \gamma)=\sigma_{0}\left(\alpha, \frac{2 \alpha}{3}\right)=\frac{2 \alpha}{3+\alpha},
$$

where

$$
\sigma_{0}(\alpha, \gamma)=\min \left(\frac{4 \alpha}{4+2 \alpha-\gamma}, \frac{4 \gamma}{4+2 \alpha-\gamma}, \frac{2(2 \alpha-\gamma)}{4+2 \alpha-\gamma}\right)
$$

This finishes the proof of Item (2) in Proposition 2.6.

Step 3. The case supp $\phi \subset\left\{x \in \bar{\Omega}, B_{0}(x)>0\right\}$.

We continue with the choice $\delta=\gamma=\frac{2}{3} \alpha$ and $\rho=4 /(4+2 \alpha-\gamma)$. We collect the inequalities in (2.13), (2.14) and Lemma 2.2 and write

$$
\begin{array}{r}
\int_{\Omega}\left|(\nabla-i \kappa H \mathbf{A})\left(\chi_{j} \phi\right)\right|^{2} d x \geq\left(1-2 \ell^{2 \alpha / 3}\right) \kappa H \int_{\Omega}\left(\Theta_{0}\left|B_{0}\left(a_{j}\right)\right|-C(\kappa H)^{-1 / 4}\right)\left|\chi_{j} \phi\right|^{2} d x \\
-C H^{2} \kappa^{-\sigma(\alpha)} \int_{\Omega}\left|\chi_{j} \phi\right|^{2} d x .
\end{array}
$$

Since $B_{0} \in C^{0, \alpha}(\bar{\Omega})$, we can replace $B_{0}\left(a_{j}\right)$ by $B_{0}(x)$ on the support of $\chi_{j}$ modulo an error $\mathcal{O}\left(\ell^{\alpha}\right)$. We insert the resulting estimate into $(2.12)$ and use that $\sum_{j} \chi_{j}^{2}=1$ to get,

$$
\int_{\Omega}|(\nabla-i \kappa H \mathbf{A}) \phi|^{2} d x \geq \kappa H \int_{\Omega}\left(\Theta_{0}\left|B_{0}(x)\right|-C\left(\kappa^{-\sigma(\alpha)}+\kappa^{-1 / 2}\right)\right)|\phi(x)|^{2} d x .
$$

Observing that $\sigma(\alpha) \leq \frac{1}{2}$, we have achieved the proof of Item (1) in Proposition 2.6.

\section{Exponential DECAY}

3.1. Main statements. We recall the definition of the de Gennes constant $\Theta_{0}$ in (1.5), and the two constants $\beta_{0}, \beta_{1}$ in (1.4). For all $\lambda \in\left(0, \beta_{0}\right)$, we introduce the two functions on $\omega(\lambda)$ :

$$
t_{\lambda}(x)=\operatorname{dist}(x, \partial \omega(\lambda)) \text { and } \zeta_{\lambda}(x)=\operatorname{dist}(x, \Omega \cap \partial \omega(\lambda)),
$$

where $\omega(\cdot)$ is the domain introduced in (1.12).

Theorem 3.1. [Exponential decay outside the superconductivity region]

Let $c_{1}$ and $c_{2}$ be two constants such that $\beta_{0}^{-1}<c_{1}<c_{2}$. Suppose that Assumption 1.2 holds for some $\alpha \in(0,1)$. There exists $\mu_{0}>0$ and for all $\mu \in\left(0, \mu_{0}\right)$, there exist $\kappa_{0}>0, C>0$ and $\hat{\alpha}>0$ such that, if

$$
\kappa \geq \kappa_{0}, \quad c_{1} \kappa \leq H \leq c_{2} \kappa,
$$

and $(\psi, \mathbf{A})_{\kappa, H}$ is a solution of (1.3), then the following inequalities hold: 
(1) Decay in the interior:

$$
\begin{aligned}
& \int_{\omega(\lambda) \cap\left\{t_{\lambda}(x) \geq \frac{1}{\sqrt{\kappa H}}\right\}}\left(|\psi(x)|^{2}+\frac{1}{\kappa H}|(\nabla-i \kappa H \mathbf{A}) \psi(x)|^{2}\right) \exp \left(2 \hat{\alpha} \sqrt{\kappa H} t_{\lambda}(x)\right) d x \leq \frac{C}{\kappa}, \\
& \text { where } \lambda=\frac{\kappa}{H}+\mu ;
\end{aligned}
$$

(2) Decay up to the boundary:

$$
\begin{aligned}
& \int_{\omega(\beta) \cap\left\{\zeta_{\beta}(x) \geq \frac{1}{\sqrt{\kappa H}}\right\}}\left(|\psi(x)|^{2}+\frac{1}{\kappa H}|(\nabla-i \kappa H \mathbf{A}) \psi(x)|^{2}\right) \exp \left(2 \hat{\alpha} \sqrt{\kappa H} \zeta_{\beta}(x)\right) d x \leq \frac{C}{\kappa}, \\
& \text { where } \beta=\Theta_{0}^{-1}\left(\frac{\kappa}{H}+\mu\right) .
\end{aligned}
$$

Remark 3.2. Theorem 3.1 says that, for $\mu>0$ sufficiently small, bulk superconductivity breaks down in the region $\left\{x \in \Omega,\left|B_{0}(x)\right| \geq \frac{\kappa}{H}+\mu\right\}$ and that surface superconductivity breaks down in the region $\left\{x \in \partial \Omega, \Theta_{0}\left|B_{0}(x)\right| \geq \frac{\kappa}{H}+\mu\right\}$. This is illustrated in Figures 1 and 2 .

Remark 3.3. In the constant magnetic field case, $B_{0}=1$, Theorem 3.1 is proved by Pan [34], in response to a conjecture by Rubinstein [37, p. 182]. Our proof of Theorem 3.1 is simpler than the one in [34] since we do not use the a priori elliptic $L^{\infty}$-estimates, whose derivation is not easy (cf. [15, Ch. 11]).

Remark 3.4. On a technical level, one can still avoid to use the $L^{\infty}$-elliptic estimates in the proof of Theorem 3.1 when the magnetic field is constant, by establishing a weak decay estimate on the order parameter (namely $\|\psi\|_{2}=\mathcal{O}\left(\kappa^{-1 / 4}\right)$ ). This has been done by Bonnaillie-Noël and Fournais in [10] and then generalized by Fournais-Helffer to non-vanishing continuous magnetic fields in [15, Cor. 12.3.2]. However, in the sign-changing field case and the regime considered in Theorem 3.1, the weak decay estimate as in [10] does not hold.

The substitute of the weak decay estimate in our proof is the use of a (local) gauge transformation. This has been used earlier to estimate the Ginzburg-Landau energy (cf. [29, 9]), and the exponential decay of the order parameter for non-smooth magnetic fields (cf. [6]). We will extend this method for obtaining local estimates in Theorems 4.7 and 4.8.

Remark 3.5. The conclusion in Theorem 1.3 is a simple consequence of Theorem 3.1 and the estimate in Proposition 2.4. Actually, if $O$ is an open set independent of $\kappa$ such that $\bar{O} \subset \omega(\kappa / H)$, then

$$
O \subset \omega\left(\frac{\kappa}{H}+\mu\right)
$$

for $\mu$ sufficiently small, and

$$
\operatorname{dist}\left(x, \partial \omega\left(\frac{\kappa}{H}+\mu\right)\right) \geq c_{\mu} \text { in } O,
$$

for a constant $c_{\mu}>0$.

Similarly, when $O$ is an open set independent of $\kappa$ and

$$
\bar{O} \subset \omega(\kappa / H) \cup\left\{x \in \partial \Omega, \Theta_{0}\left|B_{0}(x)\right|<\kappa / H\right\},
$$

then

$$
O \subset \omega\left(\Theta_{0}^{-1}\left(\frac{\kappa}{H}+\mu\right)\right)
$$

for $\mu$ sufficiently small, and

$$
\operatorname{dist}\left(x, \partial \omega\left(\Theta_{0}^{-1}\left(\frac{\kappa}{H}+\mu\right)\right)\right) \geq \hat{c}_{\mu} \quad \text { in } O,
$$

for a constant $\hat{c}_{\mu}>0$. 
The rest of this section is devoted to the proof of Theorem 3.1, which follows the scheme of the proof of the semi-classical Agmon estimates (cf. [15, Ch. 12] and references therein).

Suppose that the parameters $\kappa$ and $H$ have the same order, i.e.

$$
\kappa \geq \kappa_{0} \quad \text { and } \quad c_{1} \kappa \leq H \leq c_{2} \kappa,
$$

where $\kappa_{0} \geq 1$ is supposed sufficiently large (this condition will appear in the proof below). Suppose also that

$$
c_{2}>c_{1}>\beta_{0}^{-1}
$$

where $c_{1}, c_{2}$ are fixed constants and $\beta_{0}$ was introduced in (1.4).

3.2. Useful inequalities. For all $\gamma>0$, we extend to $\bar{\Omega}$ the definitions of $t_{\gamma}$ and $\zeta_{\gamma}$ given in (3.1) as follows

$$
t_{\gamma}(x)= \begin{cases}\operatorname{dist}(x, \partial \omega(\gamma)) & \text { if } x \in \omega(\gamma) \\ 0 & \text { if } x \in \bar{\Omega} \backslash \omega(\gamma))\end{cases}
$$

and

$$
\zeta_{\gamma}(x)=\left\{\begin{array}{ll}
\operatorname{dist}(x, \Omega \cap \partial \omega(\gamma)) & \text { if } x \in \omega(\gamma) \\
0 & \text { if } x \in \bar{\Omega} \backslash \omega(\gamma)
\end{array} .\right.
$$

In the sequel, we will add conditions on $\gamma$ to ensure that $\omega(\gamma) \neq \emptyset$.

Let $\tilde{\chi} \in C^{\infty}(\mathbb{R})$ be a non negative function satisfying

$$
\tilde{\chi}=0 \text { on }\left(-\infty, \frac{1}{2}\right], \quad \tilde{\chi}=1 \text { on }[1, \infty) .
$$

Define the functions $\chi_{\gamma}, \eta_{\gamma}, f_{\gamma}$ and $g_{\gamma}$ on $\Omega$ as follows:

$$
\begin{gathered}
\chi_{\gamma}(x)=\tilde{\chi}\left(\sqrt{\kappa H} t_{\gamma}(x)\right), \quad \eta_{\gamma}(x)=\tilde{\chi}\left(\sqrt{\kappa H} \zeta_{\gamma}(x)\right), \\
f_{\gamma}(x)=\chi_{\gamma}(x) \exp \left(\hat{\alpha} \sqrt{\kappa H} t_{\gamma}(x)\right) \quad \text { and } \quad g_{\gamma}(x)=\eta_{\gamma}(x) \exp \left(\hat{\alpha} \sqrt{\kappa H} \zeta_{\gamma}(x)\right),
\end{gathered}
$$

where $\hat{\alpha}$ is a positive number whose value will be fixed later.

Let $h \in\left\{f_{\gamma}, g_{\gamma}\right\}$. We multiply both sides of the first equation in (1.3) by $h^{2} \bar{\psi}$ and then integrate by parts over $\omega(\gamma)$. We get

$$
\int_{\omega(\gamma)}\left(|(\nabla-i \kappa H \mathbf{A})(h \psi)|^{2}-\kappa^{2} h^{2}|\psi|^{2}-|\nabla h|^{2}|\psi|^{2}\right) d x \leq 0 .
$$

In the computations below, the constant $C$ is independent of $\hat{\alpha}, \gamma, \kappa$ and $H$. We estimate the term involving $\nabla h$ as follows

$$
\int_{\omega(\gamma)}|\nabla h|^{2}|\psi|^{2} d x \leq 2 \hat{\alpha}^{2} \kappa H\|h \psi\|_{L^{2}(\omega(\lambda))}^{2}+C \kappa H T(h),
$$

where

$$
T(h):= \begin{cases}\int_{\omega(\gamma) \cap\left\{\sqrt{\kappa H} t_{\gamma}(x) \leq 1\right\}}|\psi(x)|^{2} d x \quad \text { if } h=f_{\gamma}, \\ \int_{\omega(\gamma) \cap\left\{\sqrt{\kappa H} \zeta_{\gamma}(x) \leq 1\right\}}|\psi(x)|^{2} d x \quad \text { if } h=g_{\gamma} .\end{cases}
$$

In this way we infer from (3.5) the following estimate

$$
\int_{\omega(\gamma)}\left(|(\nabla-i \kappa H \mathbf{A})(h \psi)(x)|^{2}-\kappa^{2} h(x)^{2}|\psi(x)|^{2}-2 \hat{\alpha}^{2} \kappa H h(x)^{2}|\psi(x)|^{2}\right) d x \leq C \kappa H T(h) .
$$




\subsection{Decay in the interior.}

Now we choose

$$
\gamma=\lambda=\frac{\kappa}{H}+\mu \text {. }
$$

Here $0<\mu<\mu_{0}$ and $\mu_{0}$ is sufficiently small such that $\mu_{0}+\frac{1}{c_{1}}<\beta_{0}$. This ensures that $\omega(\lambda) \neq \emptyset$.

We choose in (3.7) the function $h=f_{\lambda}$, where $f_{\lambda}$ is the function introduced in (3.4). Note that $f_{\lambda} \psi \in H_{0}^{1}(\omega(\lambda))$. We may apply the result in Proposition 2.6 to $\phi:=f_{\lambda} \psi$ and infer from (3.7)

$$
\int_{\omega(\lambda)}\left(\left(1-C \kappa^{-\sigma(\alpha)}\right)\left|B_{0}(x)\right|-2 \hat{\alpha}^{2}-\frac{\kappa}{H}\right) f_{\lambda}^{2}|\psi|^{2} d x \leq C \int_{\omega(\lambda) \cap\left\{\sqrt{\kappa H} t_{\lambda}(x) \leq 1\right\}}|\psi(x)|^{2} d x .
$$

We then use that $\left|B_{0}(x)\right| \geq \lambda$ in $\omega(\lambda)$ and that $\lambda=\frac{\kappa}{H}+\mu$. Consequently, for $0<\mu<\mu_{0}$, $0<\hat{\alpha}<\hat{\alpha}_{0}, \kappa \geq \kappa_{0}, \hat{\alpha}_{0}$ sufficiently small (for example $\hat{\alpha}_{0}^{2}<\mu / 4$ ) and $\kappa_{0}$ sufficiently large

$$
\left(1-C \kappa^{-\sigma(\alpha)}\right)\left|B_{0}(x)\right|-2 \hat{\alpha}^{2}-\frac{\kappa}{H} \geq \frac{\mu}{2} .
$$

Consequently, there exists a constant $C_{\mu}>0$ such that

$$
\begin{aligned}
\int_{\omega(\lambda)} f_{\lambda}(x)^{2}|\psi(x)|^{2} d x & \leq C_{\mu}^{-1} \int_{\omega(\lambda) \cap\left\{\sqrt{\kappa H} t_{\lambda}(x) \leq 1\right\}}|\psi(x)|^{2} d x \\
& \leq \frac{C}{\sqrt{\kappa H}} \quad \text { by }(2.8) .
\end{aligned}
$$

Inserting this into (3.7) (with $h=f_{\lambda}$ and $T\left(f_{\lambda}\right)$ defined in (3.6)) achieves the proof of Item (1) in Theorem 3.1.

3.4. Decay up to the boundary. Now we prove Item (2) in Theorem 3.1. Here we choose

$$
\gamma=\beta=\Theta_{0}^{-1}\left(\frac{\kappa}{H}+\mu\right) .
$$

Note that the estimate in Item (2) of Theorem 3.1 is trivially true if $\omega(\beta)=\emptyset$. So, we assume in the sequel that $\omega(\beta) \neq \emptyset$. This holds if

$$
H \geq c_{1} \kappa, \quad c_{1}>\left(\Theta_{0} \beta_{1}\right)^{-1},
$$

and $\mu$ is sufficiently small.

We write (3.7) for $h=g_{\beta}$, where $g_{\beta}$ is introduced in (3.4) and $T\left(g_{\beta}\right)$ in (3.6). We apply Proposition 2.6 to $\phi:=g_{\beta} \psi$ and get

$$
\int_{\omega(\beta)}\left(\left(1-C \kappa^{-\sigma(\alpha)}\right) \Theta_{0}\left|B_{0}(x)\right|-C \hat{\alpha}^{2}-\frac{\kappa}{H}\right) g_{\beta}(x)^{2}|\psi(x)|^{2} d x \leq C \int_{\omega(\beta) \cap\left\{\sqrt{\kappa H} \zeta_{\beta}(x) \leq 1\right\}}|\psi(x)|^{2} d x .
$$

We decompose the integral over $\omega(\beta)$ as follows

$$
\int_{\omega(\beta)}=\int_{\omega_{\mathrm{int}}(\beta)}+\int_{\omega_{\mathrm{bnd}}(\beta)},
$$

where

$$
\omega_{\text {int }}(\beta)=\omega(\beta) \cap\{\sqrt{\kappa H} \operatorname{dist}(x, \partial \Omega) \geq 1\} \quad \text { and } \quad \omega_{\text {bnd }}(\beta)=\omega(\beta) \cap\{\sqrt{\kappa H} \operatorname{dist}(x, \partial \Omega)<1\} .
$$

From (3.2), we see that $\zeta_{\beta}(x)=t_{\beta}(x)$ and $f_{\beta}(x)=g_{\beta}(x)$ in $\omega_{\text {int }}(\beta)$. Furthermore, from the definition of $\omega(\cdot)$ in (1.12), we see that $\omega(\beta) \subset \omega(\lambda)$ and $t_{\beta}(x) \leq t_{\lambda}(x)$ on $\omega(\beta)$ if $\beta \geq \lambda$. Hence, by the first item in Theorem 3.1 (which is already proved for all $\hat{\alpha} \in\left(0, \hat{\alpha}_{0}\right)$ ),

$$
\int_{\omega_{\text {int }}(\beta)}\left|\left(1-C \kappa^{-\sigma(\alpha)}\right) \Theta_{0}\right| B_{0}(x)\left|-2 \hat{\alpha}^{2}-\frac{\kappa}{H}\right| g_{\beta}(x)^{2}|\psi(x)|^{2} d x \leq \frac{C}{\kappa} .
$$


Thus, we infer from (3.8) (and the bound $|\psi| \leq 1$ ),

$$
\int_{\omega_{\mathrm{bnd}}(\beta)}\left(\left(1-C \kappa^{-\sigma(\alpha)}\right) \Theta_{0}\left|B_{0}(x)\right|-2 \hat{\alpha}^{2}-\frac{\kappa}{H}\right) g_{\beta}(x)^{2}|\psi(x)|^{2} d x \leq \frac{C}{\kappa} .
$$

But, in $\omega_{\text {bnd }}(\beta), \Theta_{0}\left|B_{0}(x)\right| \geq \frac{\kappa}{H}+\mu$, by definition of $\omega(\beta)$ and $\beta=\Theta_{0}^{-1}\left(\frac{\kappa}{H}+\mu\right)$. Thus, as long as $\hat{\alpha}$ is selected sufficiently small, we have

$$
\left(1-C \kappa^{-\sigma(\alpha)}\right) \Theta_{0}\left|B_{0}(x)\right|-2 \hat{\alpha}^{2}-\frac{\kappa}{H} \geq \frac{\mu}{2},
$$

and consequently, for some constant $\tilde{C}_{\mu}>0$,

$$
\int_{\omega_{\text {bnd }}(\beta)} g_{\beta}(x)^{2}|\psi(x)|^{2} d x \leq \frac{\tilde{C}_{\mu}}{\kappa} .
$$

We insert this estimate and the one in (3.9) into (3.8) to get

$$
\int_{\omega(\beta)} g_{\beta}(x)^{2}|\psi(x)|^{2} d x \leq \frac{\tilde{C}_{\mu}+C}{\kappa} .
$$

Finally, by inserting this estimate into (3.7) (with $h=g_{\beta}$ and $T\left(g_{\beta}\right)$ defined in (3.6)), we finish the proof of Item (2) in Theorem 3.1.

\section{Surface EnERGy}

The analysis of surface superconductivity starts with the work of St. James-de Gennes [38], who studied this phenomenon on the ball. In the last two decades, many papers adressed the boundary concentration of the Ginzburg-Landau order parameter for general $2 D$ and $3 D$ samples in the presence of a constant magnetic field. We refer the reader to $[3,12,14,16,19,18,31,34]$.

In this section, we study surface superconductivity in non-uniform magnetic fields. Our presentation not only generalizes the results known for the constant field case, but also provides local estimates and new proofs, see Theorems 4.7 and 4.8. The most notable novelty in the proofs is that we do not use the $L^{\infty}$ elliptic estimates.

4.1. The surface energy function. In this subsection, we give the definition of the continuous function $E_{\text {surf }}:\left[1, \Theta_{0}^{-1}\right] \rightarrow(-\infty, 0]$ introduced by X.B. Pan in [34] and which appeared after (1.14) and in Theorem 1.5. $\Theta_{0}$ is as before the de Gennes constant introduced in (1.5) with property (1.6).

For $b \in\left[1, \Theta_{0}^{-1}\right]$ and $R>0$, we consider the reduced Ginzburg-Landau functional,

$$
\mathcal{V}\left(U_{R}\right) \ni \phi \mapsto \mathcal{E}_{b, R}(\phi)=\int_{U_{R}}\left(b\left|\left(\nabla_{(\sigma, \tau)}+i \tau \mathbf{f}\right) \phi\right|^{2}-|\phi|^{2}+\frac{1}{2}|\phi|^{4}\right) d \sigma d \tau,
$$

where $\mathbf{f}=(1,0)$ and $U_{R}$ is the domain,

$$
U_{R}=(-R, R) \times(0,+\infty)
$$

and

$$
\mathcal{V}\left(U_{R}\right)=\left\{u \in L^{2}\left(U_{R}\right):\left(\nabla_{(\sigma, \tau)}+i \tau \mathbf{f}\right) u \in L^{2}\left(U_{R}\right), u( \pm R, \cdot)=0\right\} .
$$

We introduce the following ground state energy,

$$
d(b, R)=\inf \left\{\mathcal{E}_{b, R}(\phi): \phi \in \mathcal{V}\left(U_{R}\right)\right\} .
$$

In [34], it is proved that, for all $b \in\left[1, \Theta_{0}^{-1}\right]$, there exists $E_{\text {surf }}(b) \in(-\infty, 0]$ such that

$$
E_{\text {surf }}(b)=\lim _{R \rightarrow \infty} \frac{d(b, R)}{2 R} .
$$

The surface energy function $E_{\text {surf }}(\cdot)$ can be described by a simplified $1 D$ problem as well (cf. $[3,18]$ and finally [12] for the optimal result). We collect some properties of $E_{\text {surf }}(\cdot)$ :

- $E_{\text {surf }}(\cdot)$ is a continuous and increasing function (cf. [19]); 
- $E_{\text {surf }}\left(\Theta_{0}^{-1}\right)=0$ and $E_{\text {surf }}(b)<0$ for all $b \in\left[1, \Theta_{0}^{-1}\right)$ (cf. [14]).

The next theorem gives the existence of some minimizer with good properties (cf. [34, Theorems $4.4 \& 5.3])$ :

Theorem 4.1. There exist positive constants $R_{0}$ and $M$ such that, for all $b \in\left[1, \Theta_{0}^{-1}\right)$ and $R \geq R_{0}$ :

(1) The functional (4.1) has a minimizer $u_{R}$ in $\mathcal{V}\left(U_{R}\right)$ with the following properties:

(a) $u_{R} \not \equiv 0$;

(b) $\left\|u_{R}\right\|_{\infty} \leq 1$;

(c)

$$
\frac{1}{R} \int_{U_{R} \cap\{\tau \geq 3\}} \frac{\tau^{2}}{(\ln \tau)^{2}}\left(\left|\left(\nabla_{(\sigma, \tau)}+i \tau \mathbf{f}\right) u_{R}\right|^{2}+\left|u_{R}(\sigma, \tau)\right|^{2}+\tau^{2}\left|u_{R}(\sigma, \tau)\right|^{4}\right) d \sigma d \tau \leq M .
$$

(2) The surface energy function $E_{\text {surf }}(b)$ satisfies

$$
E_{\text {surf }}(b) \leq \frac{d(b, R)}{2 R} \leq E_{\text {surf }}(b)+\frac{M}{R} .
$$

The upper bound in Item (2) above results from a property of superadditivity of $d(b, R)$, see [34, Eq. (5.4)]. The lower bound in Item (2) above is not explicitly mentioned in [34], but its derivation is easy [17, Proof of Thm 2.1, Step 2, p. 351] and can be sketched in the following way. Let $R>0$ and $n \in \mathbb{N}$. Let $u_{R} \in H_{0}^{1}\left(U_{R}\right)$ be a minimizer of the functional in (4.1). We extend $u_{R}$ to a function in $H_{0}^{1}\left(U_{(2 n+1) R}\right)$ by periodicity as follows

$$
u_{R}\left(x_{1}+2 R, x_{2}\right)=u_{R}\left(x_{1}, x_{2}\right) .
$$

Consequently,

$$
d(b,(2 n+1) R) \leq \mathcal{E}_{b,(2 n+1) R}\left(u_{R}\right)=(2 n+1) d(b, R) .
$$

Dividing both sides of the preceding inequality by $2(2 n+1) R$ and sending $n$ to $+\infty$, we get

$$
E_{\text {surf }}(b) \leq \frac{d(b, R)}{2 R} .
$$

4.2. Boundary coordinates. The analysis of the boundary effects is performed in specific coordinates valid in a tubular neighborhood of $\partial \Omega$. We call these coordinates boundary coordinates. For more details on these coordinates, see for instance [15, Appendix F].

For a sufficiently small $t_{0}>0$, we introduce the open set

$$
\Omega\left(t_{0}\right)=\left\{x \in \mathbb{R}^{2}: \operatorname{dist}(x, \partial \Omega)<t_{0}\right\} .
$$

In the sequel, let $x_{0} \in \partial \Omega$ be a fixed point. Let $s \mapsto \gamma_{x_{0}}(s)$ be the parametrization of $\partial \Omega$ by arc-length such that $\gamma_{x_{0}}(0)=x_{0}$. Also, let $\nu(s)$ be the unit inward normal of $\partial \Omega$ at $\gamma_{x_{0}}(s)$. The orientation of $\gamma_{x_{0}}$ is selected in the counter clock-wise direction, hence

$$
\operatorname{det}\left(\gamma_{x_{0}}^{\prime}(s), \nu(s)\right)=1 \text {. }
$$

Define the transformation

$$
\Phi_{x_{0}}:\left[-\frac{|\partial \Omega|}{2}, \frac{|\partial \Omega|}{2}\right) \times\left(0, t_{0}\right) \ni(s, t) \mapsto \gamma_{x_{0}}(s)+t \nu(s) \in \Omega\left(t_{0}\right) .
$$

We may choose $t_{0}$ sufficiently small (independently from the choice of the point $x_{0} \in \partial \Omega$ ) such that the transformation in (4.6) is a diffeomorphism. The Jacobian of this transformation is $\left|D \Phi_{x_{0}}\right|=1-t k(s)$, where $k$ denotes the curvature of $\partial \Omega$. For $x \in \Omega\left(t_{0}\right)$, we put

$$
\Phi_{x_{0}}^{-1}(x)=(s(x), t(x)) .
$$

In particular, we get the explicit formulae

$$
t(x)=\operatorname{dist}(x, \partial \Omega) \quad \text { and } \quad s\left(x_{0}\right)=0 .
$$


Using $\Phi_{x_{0}}$, we may associate to any function $u \in L^{2}(\Omega)$, a function $\widetilde{u}=T_{\Phi_{x_{0}}} u$ defined in $\left[-\frac{|\partial \Omega|}{2}, \frac{|\partial \Omega|}{2}\right) \times\left(0, t_{0}\right)$ by

$$
\widetilde{u}(s, t)=u\left(\Phi_{x_{0}}(s, t)\right) .
$$

Also, for every vector field $\mathbf{A} \in H^{1}(\Omega)$, we assign the vector field

$$
\tilde{\mathbf{A}}(s, t)=\left(\tilde{\mathbf{A}}_{1}(s, t), \tilde{\mathbf{A}}_{2}(s, t)\right)
$$

with

and

$$
\left\{\begin{array}{l}
\tilde{\mathbf{A}}_{1}(s, t)=a(s, t) \mathbf{A}\left(\Phi_{x_{0}}(s, t)\right) \cdot \gamma_{x_{0}}^{\prime}(s) \\
\tilde{\mathbf{A}}_{2}(s, t)=\mathbf{A}\left(\Phi_{x_{0}}(s, t)\right) \cdot \nu(s)
\end{array}\right.
$$

The following change of variable formulas hold.

Proposition 4.2. For $u \in H^{1}(\Omega)$ and $\mathbf{A} \in H^{1}\left(\Omega ; \mathbb{R}^{2}\right)$, we have:

$$
\int_{\Omega\left(t_{0}\right)}|(\nabla-i \mathbf{A}) u|^{2} d x=\int_{0}^{t_{0}} \int_{-\frac{|\partial \Omega|}{2}}^{\frac{|\partial \Omega|}{2}}\left[[a(s, t)]^{-2}\left|\left(\partial_{s}-i \tilde{\mathbf{A}}_{1}\right) \widetilde{u}\right|^{2}+\left|\left(\partial_{t}-i \tilde{\mathbf{A}}_{2}\right) \widetilde{u}\right|^{2}\right] a(s, t) d s d t,
$$

and

$$
\int_{\Omega\left(t_{0}\right)}|u(x)|^{2} d x=\int_{0}^{t_{0}} \int_{-\frac{|\partial \Omega|}{2}}^{\frac{|\partial \Omega|}{2}}|\widetilde{u}(s, t)|^{2} a(s, t) d s d t .
$$

Recall the vector field $\mathbf{A}_{0}$ introduced in (2.2). Up to a gauge transformation, the vector field $\mathbf{A}_{0}$ admits a useful (local) representation in the coordinate system $(s, t)$.

For $x_{0} \in \partial \Omega$ and $\ell \in\left(0, t_{0}\right)$, we introduce the set $V_{x_{0}}(\ell) \subset \Omega\left(t_{0}\right)$ as follows:

$$
V_{x_{0}}(\ell)=\Phi_{x_{0}}((-\ell, \ell) \times(0, \ell)) .
$$

Lemma 4.3. There exists $r_{0}>0$ such that, for any $x_{0}$ in $\partial \Omega$, there exists $g_{x_{0}}$ in $C^{\infty}\left(\left(-2 r_{0}, 2 r_{0}\right) \times\left(0, r_{0}\right)\right)$ such that

$$
\tilde{\mathbf{A}}_{0}(s, t)-\nabla g_{x_{0}}(s, t)=\left(-t+k(s) \frac{t^{2}}{2}, 0\right) \quad \text { in }\left(-2 r_{0}, 2 r_{0}\right) \times\left(0, r_{0}\right) .
$$

Here $\tilde{\mathbf{A}}_{0}$ is the vector field associated with $\mathbf{A}_{0}$ by the formulas in (4.9) and one can take $r_{0}=$ $\min \left(t_{0}, \frac{|\partial \Omega|}{4}\right)$.

For the proof of Lemma 4.3, we refer to [15, Proof of Lem. F.1.1]. Note that Lemma F.1.1 in $[15]$ is announced for a more general setting.

We will use Lemma 4.3 to estimate the following Ginzburg-Landau energy of $u$,

$$
\mathcal{G}_{0}\left(u, \mathbf{A}_{0} ; V_{x_{0}}(\ell)\right)=\int_{V_{x_{0}}(\ell)}\left(\left|\left(\nabla-i h_{\mathrm{ex}} \mathbf{A}_{0}\right) u\right|^{2}-\kappa^{2}|u|^{2}+\frac{\kappa^{2}}{2}|u|^{4}\right) d x .
$$

Lemma 4.4. There exist constants $C>0, \ell_{0}>0$ and $\kappa_{0}>0$ such that, for all $x_{0} \in \partial \Omega$, $\ell \in\left(0, \ell_{0}\right), \kappa \geq \kappa_{0}, \kappa^{2} \leq h_{\mathrm{ex}} \leq \Theta_{0}^{-1} \kappa^{2}$, and $u \in H_{0}^{1}\left(V_{x_{0}}(\ell)\right) \cap L^{\infty}\left(V_{x_{0}}(\ell)\right)$ satisfying $\|u\|_{\infty} \leq 1$, the following two inequalities hold:

$$
\mathcal{G}_{0}\left(u, \mathbf{A}_{0} ; V_{x_{0}}(\ell)\right) \geq 2 \frac{\kappa^{2} \ell}{\sqrt{h_{\mathrm{ex}}}} E_{\mathrm{surf}}\left(\frac{h_{\mathrm{ex}}}{\kappa^{2}}\right)-C \kappa \ell\left(\ell+\kappa^{3} \ell^{4}+\kappa \ell^{2}\right),
$$

and

$$
\mathcal{G}_{0}\left(u, \mathbf{A}_{0} ; V_{x_{0}}(\ell)\right) \leq(1+C \ell) \frac{\kappa^{2}}{h_{\mathrm{ex}}} \mathcal{E}_{h_{\mathrm{ex}} / \kappa^{2}, \sqrt{h_{\mathrm{ex}}} \ell}(\widetilde{v})+C \kappa \ell\left(\kappa^{3} \ell^{4}+\kappa \ell^{2}\right) .
$$


where $\mathcal{E}_{.,}$is the functional introduced in (4.1) and

$$
\widetilde{v}(\sigma, \tau)=\exp \left(-i h_{\mathrm{ex}} g_{x_{0}}\left(\frac{\sigma}{\sqrt{h_{\mathrm{ex}}}}, \frac{\tau}{\sqrt{h_{\mathrm{ex}}}}\right)\right) \widetilde{u}\left(\frac{\sigma}{\sqrt{h_{\mathrm{ex}}}}, \frac{\tau}{\sqrt{h_{\mathrm{ex}}}}\right) .
$$

Here $\widetilde{u}$ is the function associated with $u$ by (4.8) and $g_{x_{0}}$ is introduced in Lemma 4.3.

Proof. Using Proposition 4.2 and the assumptions on $u$, we may write, for two constants $C_{0}, C>$ 0 and for all $0<\ell<\min \left(\frac{1}{2} C_{0}^{-1}, t_{0}\right)$,

$$
\mathcal{G}_{0}\left(u, \mathbf{A}_{0} ; V_{x_{0}}(\ell)\right) \geq\left(1-C_{0} \ell\right) \int_{0}^{\ell} \int_{-\ell}^{\ell}\left(\left|\left(\nabla-i h_{\mathrm{ex}} \widetilde{\mathbf{A}}_{0}\right) \widetilde{u}\right|^{2}-\kappa^{2}|\widetilde{u}|^{2}+\frac{\kappa^{2}}{2}|\widetilde{u}|^{4}\right) d s d t-C \kappa^{2} \ell^{3} .
$$

Let $g:=g_{x_{0}}$ be the function defined in Lemma 4.3 and $\widetilde{w}(s, t)=e^{-i h_{\mathrm{ex}} g(s, t)} \widetilde{u}(s, t)$. Using the Cauchy-Schwarz inequality, we get the existence of $C>0$ such that

$\mathcal{G}_{0}\left(u, \mathbf{A}_{0} ; V_{x_{0}}(\ell)\right) \geq\left(1-2 C_{0} \ell\right) \int_{0}^{\ell} \int_{-\ell}^{\ell}\left(\left|\left(\nabla+i h_{\mathrm{ex}} t \mathbf{f}\right) \widetilde{w}\right|^{2}-\kappa^{2}|\widetilde{w}|^{2}+\frac{\kappa^{2}}{2}|\widetilde{w}|^{4}\right) d s d t-C \kappa^{4} \ell^{5}-C \kappa^{2} \ell^{3}$.

Here $\mathbf{f}=(1,0)$. We apply the change of variables $(\sigma, \tau)=\left(\sqrt{h_{\mathrm{ex}}} s, \sqrt{h_{\mathrm{ex}}} t\right)$ and $\widetilde{v}(\sigma, \tau)=\widetilde{w}(s, t)$ to get

$$
\mathcal{G}_{0}\left(u, \mathbf{A}_{0} ; V_{x_{0}}(\ell)\right) \geq\left(1-2 C_{0} \ell\right) \frac{\kappa^{2}}{h_{\mathrm{ex}}} \mathcal{E}_{h_{\mathrm{ex}} / \kappa^{2}, R}(\widetilde{v})-C \kappa^{4} \ell^{5}-C \kappa^{2} \ell^{3},
$$

where $R=h_{\mathrm{ex}}^{\frac{1}{2}} \ell$ and $\mathcal{E}_{h_{\mathrm{ex}} / \kappa^{2}, R}$ is the functional introduced in (4.1) for $b=h_{\mathrm{ex}} / \kappa^{2}$.

Note that we extended $\widetilde{v}$ by 0 , which is possible because $u \in H_{0}^{1}\left(V_{x_{0}}(\ell)\right)$. Using the second Item in Theorem 4.1 and the assumption $C_{0} \ell<\frac{1}{2}$, we get

$$
\mathcal{G}_{0}\left(u, \mathbf{A}_{0} ; V_{x_{0}}(\ell)\right) \geq 2\left(1-2 C_{0} \ell\right) \frac{\kappa^{2}}{h_{\mathrm{ex}}}\left(h_{\mathrm{ex}}^{\frac{1}{2}} \ell\right) E_{\mathrm{surf}}\left(\frac{h_{\mathrm{ex}}}{\kappa^{2}}\right)-C \kappa^{4} \ell^{5}-C \kappa^{2} \ell^{3} .
$$

This proves the lower bound (4.14) in Lemma 4.4.

Similarly, using Lemma 4.3, the Cauchy-Schwarz inequality on the kinetic term and a change of variables, we get the upper bound (4.15) of Lemma 4.4.

\subsection{Existence of surface superconductivity.}

The proof of Theorem 1.5 follows from the exponential decay stated in Theorem 3.1 and the following result:

Theorem 4.5. Suppose that Assumption 1.2 holds and that $b>\beta_{0}^{-1}$, where $\beta_{0}$ is the constant introduced in (1.4). There exists $\rho \in(0,1)$ such that the following is true.

Let $x_{0} \in \partial \Omega$ such that $\frac{1}{b}<\left|B_{0}\left(x_{0}\right)\right|<\frac{1}{\Theta_{0} b}$. If $(\psi, \mathbf{A})_{\kappa, H}$ is a minimizer of the functional in (1.1) for $H=b \kappa$, then

$$
\lim _{\kappa \rightarrow+\infty}\left(2 \kappa^{1+\rho} \int_{V_{x_{0}}\left(\kappa^{-\rho}\right)}|\psi(x)|^{4} d x\right)=-2 \sqrt{\frac{1}{b\left|B_{0}\left(x_{0}\right)\right|}} E_{\text {surf }}\left(b\left|B_{0}\left(x_{0}\right)\right|\right)>0,
$$

and

$$
\lim _{\kappa \rightarrow+\infty}\left(2 \kappa^{\rho-1} \mathcal{E}\left(\psi, \mathbf{A} ; V_{x_{0}}\left(\kappa^{-\rho}\right)\right)\right)=\sqrt{\frac{1}{b\left|B_{0}\left(x_{0}\right)\right|}} E_{\text {surf }}\left(b\left|B_{0}\left(x_{0}\right)\right|\right)<0 .
$$

The proof of Theorem 4.5 will follow from the upper bound in Theorems 4.7 and 4.8 below. Remark 4.6. Let $\epsilon \in\left(1, \Theta_{0}^{-1}-1\right)$. The convergence in (4.16) and (4.17) is uniform with respect to $x_{0} \in\left\{1+\epsilon \leq b\left|B_{0}\right|<\Theta_{0}^{-1}\right\} \cap \partial \Omega$. This is precisely stated in Theorems 4.7 and 4.8. 


\subsection{Sharp upper bound on the $L^{4}$-norm.}

In this subsection, we will prove:

Theorem 4.7. Suppose that $B_{0} \in C^{0, \alpha}(\bar{\Omega})$ for some $\alpha \in(0,1), \rho \in\left(\frac{3}{3+\alpha}, 1\right)$ and

$$
b \geq \beta_{0}^{-1}, \text { with } \beta_{0}:=\sup _{x \in \bar{\Omega}}\left|B_{0}(x)\right|>0 .
$$

There exist $\kappa_{0}>0$, a function $\mathrm{r}:\left[\kappa_{0},+\infty\right) \rightarrow \mathbb{R}_{+}$such that $\lim _{\kappa \rightarrow+\infty} \mathrm{r}(\kappa)=0$ and, for all $\kappa \geq \kappa_{0}$, for all critical point $(\psi, \mathbf{A})_{\kappa, H}$ of the functional in (1.1) with $H=b \kappa$, and all $x_{0} \in \partial \Omega$ satisfying

$$
1 \leq b\left|B_{0}\left(x_{0}\right)\right|<\Theta_{0}^{-1}
$$

the inequality

$$
\frac{1}{2 \ell} \int_{V_{x_{0}}(\ell)}|\psi(x)|^{4} d x \leq-2 \kappa^{-1} \sqrt{\frac{1}{b\left|B_{0}\left(x_{0}\right)\right|}} E_{\text {surf }}\left(b\left|B_{0}\left(x_{0}\right)\right|\right)+\kappa^{-1} \mathrm{r}(\kappa),
$$

holds with

Proof.

$$
\ell=\kappa^{-\rho} \text { and } \quad V_{x_{0}}(\ell) \text { is defined in (4.12). }
$$

The proof is reminiscent of the method used by the second author in [26, Sec. 4] (see also [27]). We assume that $B_{0}\left(x_{0}\right)>0$. The case where $B_{0}\left(x_{0}\right)<0$ can be treated in the same manner by applying the transformation $u \mapsto \bar{u}$.

Let $\sigma \in(0,1)$ and $\ell=\kappa^{-\rho}$ as in the statement of Theorem 4.7. Let $f$ be a smooth function satisfying,

$$
f=1 \quad \text { in } V_{x_{0}}(\ell), \quad 0 \leq f \leq 1 \text { and }|\nabla f| \leq \frac{C}{\sigma \ell} \text { in } V_{x_{0}}((1+\sigma) \ell) .
$$

The function $f$ depends on the parameters $x_{0}, \ell, \sigma$ but the constant $C$ is independent of these parameters. We will estimate the following local energy

$$
\mathcal{E}_{1}(f \psi, \mathbf{A}):=\mathcal{E}_{1}\left(f \psi, \mathbf{A} ; V_{x_{0}}((1+\sigma) \ell)\right),
$$

where, for an open set $\mathcal{V} \subset \Omega$,

$$
\begin{gathered}
\mathcal{E}_{1}(u, \mathbf{A} ; \mathcal{V}):=\int_{\mathcal{V}}\left(|(\nabla-i \kappa H \mathbf{A}) u|^{2}-\kappa^{2}|u|^{2}+\frac{\kappa^{2}}{2}|u|^{4}\right) d x \\
\mathcal{E}_{2}(u, \mathbf{A} ; \mathcal{V}):=\int_{\mathcal{V}}\left(|(\nabla-i \kappa H \mathbf{A}) u|^{2}-\kappa^{2}|u|^{2}+\frac{\kappa^{2}}{2}|u|^{4}\right) d x \\
+(\kappa H)^{2} \int_{\Omega}\left|\operatorname{curl} \mathbf{A}-B_{0}\right|^{2} d x .
\end{gathered}
$$

Since $(\psi, \mathbf{A})$ is a solution of (1.3), an integration by parts yields (cf. [16, Eq. (6.2)]),

$$
\mathcal{E}_{1}(f \psi, \mathbf{A})=\kappa^{2} \int_{V_{x_{0}}((1+\sigma) \ell)} f^{2}\left(-1+\frac{1}{2} f^{2}\right)|\psi|^{4} d x+\int_{V_{x_{0}}((1+\sigma) \ell)}|\nabla f|^{2}|\psi|^{2} d x .
$$

Since $f=1$ in $V_{x_{0}}(\ell)$ and $-1+\frac{1}{2} f^{2} \leq-\frac{1}{2}$ in $V_{x_{0}}((1+\sigma) \ell)$, we may write

$$
\int_{V_{x_{0}}((1+\sigma) \ell)} f^{2}\left(-1+\frac{1}{2} f^{2}\right)|\psi|^{4} d x \leq-\frac{1}{2} \int_{V_{x_{0}}(\ell)}|\psi|^{4} d x .
$$

We estimate the integral in (4.21) involving $|\nabla f|$ using (4.18) and $|\operatorname{supp} f| \leq C \sigma \ell^{2}$, where $|\operatorname{supp} f|$ denotes the area of the support of $f$. In this way, we infer from (4.21),

$$
\mathcal{E}_{1}(f \psi, \mathbf{A}) \leq-\frac{\kappa^{2}}{2} \int_{V_{x_{0}}(\ell)}|\psi|^{4} d x+C \sigma^{-1} .
$$


Now we write a lower bound for this energy. We may find a real-valued function $w \in C^{2, \alpha}\left(\overline{V_{x_{0}}((1+\sigma) \ell}\right)$ such that

$$
\begin{array}{r}
\mathcal{E}_{1}(f \psi, \mathbf{A}) \geq \int_{V_{x_{0}}((1+\sigma) \ell)}\left(\left(1-C \ell^{\delta}\right)\left|\left(\nabla-i \kappa H B_{0}\left(x_{0}\right) \mathbf{A}_{0}\right)\left(e^{-i \kappa H w} f \psi\right)\right|^{2}-\kappa^{2}|f \psi|^{2}+\frac{\kappa^{2}}{2}|f \psi|^{4}\right) d x \\
-C \kappa^{2}\left(\ell^{2 \gamma-\delta}+\kappa^{2} \ell^{2+2 \alpha-\delta}\right) \int_{V_{x_{0}}((1+\sigma) \ell)}|f \psi|^{2}
\end{array}
$$

where $\gamma \in(0,1)$ is a constant whose choice will be specified later and $\delta>0$.

The details of these computations are given in (2.13) and (2.14).

From now on we choose $\delta=\alpha$, use the lower bound in Lemma 4.4 and the assumption that $H=b \kappa$ to write

$$
\begin{gathered}
\mathcal{E}_{1}(f \psi, \mathbf{A}) \geq 2\left(1-C \ell^{\alpha}\right) \kappa(1+\sigma) \ell \sqrt{\frac{1}{b\left|B_{0}\left(x_{0}\right)\right|}} E_{\text {surf }}\left(b\left|B_{0}\left(x_{0}\right)\right|\right) \\
-C \kappa \ell\left(\ell+\kappa^{3} \ell^{4}+\kappa \ell^{2}\right)-C \kappa^{2}\left(\ell^{\alpha}+\ell^{2 \gamma-\alpha}+\kappa^{2} \ell^{2+\alpha}\right) \int_{V_{x_{0}}((1+\sigma) \ell)}|f \psi|^{2} .
\end{gathered}
$$

Using the bound $\|f \psi\|_{\infty} \leq 1$, we get further

$$
\begin{aligned}
\mathcal{E}_{1}(f \psi, \mathbf{A}) \geq 2\left(1-C \ell^{\alpha}\right) \kappa(1+\sigma) \ell \sqrt{\frac{1}{b\left|B_{0}\left(x_{0}\right)\right|}} & E_{\text {surf }}\left(b\left|B_{0}\left(x_{0}\right)\right|\right) \\
- & C \kappa \ell\left(\ell+\kappa \ell^{1+\alpha}+\kappa \ell^{1+2 \gamma-\alpha}+\kappa^{3} \ell^{3+\alpha}\right) .
\end{aligned}
$$

To optimize the remainder, we choose $\gamma=\alpha$. Our assumption

$$
\ell=\kappa^{-\rho} \text { with }(1+\alpha)^{-1}<3(3+\alpha)^{-1}<\rho<1
$$

yields that the function

$$
\Sigma(\kappa, \ell):=\ell^{\alpha}+\ell+\kappa \ell^{1+\alpha}+\kappa^{3} \ell^{3+\alpha}
$$

tends, with $\ell=\kappa^{-\rho}$, to 0 as $\kappa \rightarrow+\infty$.

Now, coming back to (4.22), we find

$$
2 \kappa(1+\sigma) \ell \sqrt{\frac{1}{b\left|B_{0}\left(x_{0}\right)\right|}} E_{\text {surf }}\left(b\left|B_{0}\left(x_{0}\right)\right|\right)-C \kappa \ell \Sigma(\kappa, \ell) \leq-\frac{\kappa^{2}}{2} \int_{V_{x_{0}}(\ell)}|\psi|^{4} d x+C \sigma^{-1} .
$$

We rearrange the terms in this inequality, divide by $\kappa^{2} \ell$, and choose $\sigma=\kappa^{\frac{1}{2}(\rho-1)}$. In this way, we get the upper bound in Theorem 4.7 with, for some constant $C>0$,

$$
\mathrm{r}(\kappa)=C\left(\Sigma\left(\kappa, \kappa^{-\rho}\right)+\kappa^{\frac{1}{2}(\rho-1)}\right) .
$$

\subsection{Sharp Lower bound on the $L^{4}$-norm.}

In this subsection, we will prove the asymptotic optimality of the upper bound established in Theorem 4.7 by giving a lower bound with the same asymptotics.

We remind the reader of the definition of the domain $V_{x_{0}}(\ell)$ in (4.12) and the local energy $\mathcal{E}_{1}(\psi, \mathbf{A} ; \mathcal{V})$ introduced in (4.20).

Theorem 4.8. Let $1<\epsilon<\Theta_{0}^{-1}-1, \frac{3}{3+\alpha}<\rho<1$ and $1-\rho<\delta<1$ be constants. Under the assumptions of Theorem 4.7, there exist $\kappa_{0}>0$, a function $\hat{\mathrm{r}}:\left[\kappa_{0},+\infty\right) \rightarrow \mathbb{R}_{+}$such that $\lim _{\kappa \rightarrow+\infty} \hat{\mathrm{r}}(\kappa)=0$ and, for all $\kappa \geq \kappa_{0}$, for all minimizer $(\psi, \mathbf{A})_{\kappa, H}$ of the functional in (1.1) with $H=b \kappa$, and all $x_{0} \in \partial \Omega$ satisfying

$$
1+\epsilon \leq b\left|B_{0}\left(x_{0}\right)\right|<\Theta_{0}^{-1}
$$


the two inequalities

$$
\begin{aligned}
& \frac{1}{2 \ell} \int_{V_{x_{0}}(\ell)}|\psi(x)|^{4} d x \geq-2 \kappa^{-1} \sqrt{\frac{1}{b\left|B_{0}\left(x_{0}\right)\right|}} E_{\text {surf }}\left(b\left|B_{0}\left(x_{0}\right)\right|\right)-\kappa^{-1} \hat{\mathrm{r}}(\kappa), \\
& \left|\frac{1}{2 \ell} \mathcal{E}_{1}\left(\psi, \mathbf{A} ; V_{x_{0}}((1+\sigma) \ell)\right)-\kappa \sqrt{\frac{1}{b\left|B_{0}\left(x_{0}\right)\right|}} E_{\text {surf }}\left(b\left|B_{0}\left(x_{0}\right)\right|\right)\right| \leq \kappa \hat{\mathrm{r}}(\kappa),
\end{aligned}
$$

hold, with $\ell=\kappa^{-\rho}$ and $\sigma=\kappa^{-\delta}$.

Remark 4.9. Let $c_{2}>c_{1}>0$ be fixed constants. The conclusion in Theorem 4.8 remains true if $\ell$ satisfies

$$
c_{1} \kappa^{-\rho} \leq \ell \leq c_{2} \kappa^{-\rho} .
$$

Proof of Theorem 4.8. In the sequel, $\sigma \in(0,1)$ will be selected as a negative power of $\kappa, \sigma=\kappa^{-\delta}$ for a suitable constant $\delta \in(0,1)$. As the proof of Theorem 4.7, we can assume that $B_{0}\left(x_{0}\right)>0$. The proof of the lower bound in Theorem 4.8 will be done in four steps.

\section{Step 1: Construction of a trial function.}

The construction of the trial function here is reminiscent of that by Sandier-Serfaty in the study of bulk superconductivity (cf. [40]). Define the function

$$
u(x)=\mathbf{1}_{V_{x_{0}}((1+\sigma) \ell)}(x) \chi\left(\frac{t(x)}{\ell}\right) \exp (i \kappa H w(x)) v_{R} \circ \Phi_{x_{0}}^{-1}(x)+\eta_{\ell}(x) \psi(x) \quad(x \in \Omega) .
$$

Here $V_{x_{0}}(\cdot)$ is introduced in $(4.12), t(x)=\operatorname{dist}(x, \partial \Omega), \Phi_{x_{0}}$ is the coordinate transformation defined in (4.6),

and (cf. (4.1))

$$
\begin{gathered}
v_{R}(s, t)=\exp \left(i \kappa H g_{x_{0}}(s, t)\right) u_{R}\left(s \sqrt{B_{0}\left(x_{0}\right) \kappa H}, t \sqrt{B_{0}\left(x_{0}\right) \kappa H}\right), \\
R=(1+\sigma) \ell \sqrt{B_{0}\left(x_{0}\right) \kappa H},
\end{gathered}
$$

$u_{R}(\cdot)$ is a minimizer of the reduced functional $\mathcal{E}_{b B_{0}\left(x_{0}\right), R}(\cdot)$.

The function $g_{x_{0}}(s, t)$ satisfies the following identity in $(-2 \ell, 2 \ell) \times(0, \ell)$ (cf. Lemma 4.3),

$$
\tilde{\mathbf{A}}_{0}(s, t)-\nabla g_{x_{0}}(s, t)=\left(-t+\frac{t^{2}}{2} k(s), 0\right) .
$$

The function $\chi \in C^{\infty}([0, \infty))$ satisfies

$$
\chi=1 \text { in }[0,1 / 2], \quad \chi=0 \text { in }[1, \infty), \text { and } 0 \leq \chi \leq 1 .
$$

The function $\eta_{\ell}$ is a smooth function satisfying

$$
\eta_{\ell}(x)=0 \text { in } V_{x_{0}}((1+\sigma) \ell), \quad \eta_{\ell}(x)=1 \text { in } \Omega \backslash V_{x_{0}}((1+2 \sigma) \ell), \quad 0 \leq \eta_{\ell}(x) \leq 1 \text { in } \Omega,
$$

and

for some constant $C>0$.

$$
\left|\nabla \eta_{\ell}(x)\right| \leq C \sigma^{-1} \ell^{-1} \quad \text { in } \Omega
$$

Finally, the function $w$ is the sum of two real-valued $C^{2, \alpha}$-functions $w_{1}$ and $w_{2}$ in $V_{x_{0}}((1+\sigma) \ell)$ and satisfying the following estimates

$$
\left|\mathbf{A}(x)-\mathbf{F}(x)-\nabla w_{1}(x)\right| \leq \frac{C}{\kappa} \ell^{\alpha} \quad \text { and } \quad\left|\mathbf{F}(x)-B_{0}\left(x_{0}\right) \mathbf{A}_{0}(x)-\nabla w_{2}(x)\right| \leq C \ell^{1+\alpha} \quad \text { in } V_{x_{0}}((1+\sigma) \ell) .
$$

By Proposition 2.4, we simply define $w_{1}(x)=\left(x-x_{0}\right) \cdot\left(\mathbf{A}\left(x_{0}\right)-\mathbf{F}\left(x_{0}\right)\right)$. The fact that the vector field $\mathbf{A}_{0}(x)$ is gauge equivalent to $\mathbf{A}_{0}\left(x-x_{0}\right)$ and Lemma 2.5 ensure the existence of $w_{2}$.

We decompose the energy $\mathcal{E}(u, \mathbf{A})$ as follows

$$
\mathcal{E}(u, \mathbf{A})=\mathcal{E}_{1}(u, \mathbf{A})+\mathcal{E}_{2}(u, \mathbf{A})
$$


where

$$
\mathcal{E}_{1}(u, \mathbf{A})=\mathcal{E}_{1}\left(u, \mathbf{A} ; V_{x_{0}}((1+\sigma) \ell)\right) \quad \text { and } \quad \mathcal{E}_{2}(u, \mathbf{A})=\mathcal{E}_{2}\left(u, \mathbf{A} ; \Omega \backslash V_{x_{0}}((1+\sigma) \ell)\right)
$$

are introduced in (4.20).

Step 2: Estimating $\mathcal{E}_{1}(u, \mathbf{A})$.

Using the Cauchy-Schwarz inequality and the estimates in (4.27), we get

$$
\mathcal{E}_{1}(u, \mathbf{A}) \leq\left(1+\ell^{\alpha}\right) \mathcal{E}_{1}\left(e^{-i \kappa H w} u, B_{0}\left(x_{0}\right) \mathbf{A}_{0}\right)+C\left(\kappa^{2} \ell^{2+\alpha}+\kappa^{4} \ell^{4+\alpha}\right) .
$$

For estimating the term $\mathcal{E}_{1}\left(e^{-i \kappa H w} u, B_{0}\left(x_{0}\right) \mathbf{A}_{0}\right)$, we write

$$
\mathcal{E}_{1}\left(e^{-i \kappa H w} u, B_{0}\left(x_{0}\right) \mathbf{A}_{0}\right)=\mathcal{G}_{0}\left(e^{-i \kappa H w} u, h_{\mathrm{ex}} \mathbf{A}_{0} ; V_{x_{0}}(\tilde{\ell})\right)
$$

where

$$
\tilde{\ell}=(1+\sigma) \ell, \quad h_{\mathrm{ex}}=\kappa H B_{0}\left(x_{0}\right) \quad \text { and } \mathcal{G}_{0} \text { is introduced in }(4.13) .
$$

We apply Lemma 4.4 and get

$$
\mathcal{E}_{1}(u, \mathbf{A}) \leq\left(1+C \ell^{\alpha}\right) \frac{1}{b B_{0}\left(x_{0}\right)} \mathcal{E}_{b B_{0}\left(x_{0}\right), R}\left(\tilde{\chi}_{\ell} u_{R}\right)+C \kappa \ell\left(\kappa^{3} \ell^{3+\alpha}+\kappa \ell^{1+\alpha}\right),
$$

where

$$
\tilde{\chi}_{\ell}(\tau)=\chi\left(\frac{\tau}{\ell \sqrt{\kappa H}}\right), b=H / \kappa, \text { and } R=\sqrt{h_{\mathrm{ex}}} \tilde{\ell},
$$

in conformity with (4.26).

Note that $\operatorname{supp}\left(1-\widetilde{\chi}_{\ell}^{2}\right) \subset[\ell \sqrt{\kappa H} / 2,+\infty)$ and $\operatorname{supp} \widetilde{\chi}_{\ell}^{\prime} \subset[\ell \sqrt{\kappa H} / 2, \ell \sqrt{\kappa H}]$. Using the decay of $u_{R}$ established in Theorem 4.1, we get

$$
\mathcal{E}_{b B_{0}\left(x_{0}\right), R}\left(\widetilde{\chi}_{\ell} u_{R}\right) \leq \mathcal{E}_{b B_{0}\left(x_{0}\right), R}\left(u_{R}\right)+C \frac{|\ln (\ell \sqrt{\kappa H})|^{2}}{\ell \sqrt{\kappa H}} .
$$

Since $\mathcal{E}_{b B_{0}\left(x_{0}\right), R}\left(u_{R}\right)=d\left(b B_{0}\left(x_{0}\right), R\right)$ and $R=(1+\sigma) \ell \sqrt{B_{0}\left(x_{0}\right) \kappa H}$, Theorem 4.1 yields

$$
\begin{aligned}
\mathcal{E}_{1}(u, \mathbf{A}) \leq 2 \kappa \ell \sqrt{\frac{1}{b\left|B_{0}\left(x_{0}\right)\right|}} & E_{\text {surf }}\left(b B_{0}\left(x_{0}\right)\right) \\
+ & C \kappa \ell\left(\ell^{\alpha}+\kappa^{3} \ell^{3+\alpha}+\kappa \ell^{1+\alpha}+\sigma+(\kappa \ell)^{-1}+\frac{|\ln (\ell \sqrt{\kappa H})|^{2}}{\ell^{2} \kappa^{2}}\right) .
\end{aligned}
$$

Step 3: Estimating $\mathcal{E}_{2}(u, \mathbf{A})$.

Let $V_{x_{0}}(\tilde{\ell})^{\complement}:=\Omega \backslash V_{x_{0}}(\tilde{\ell})$ and $u=\eta_{\ell} \psi$. By the Cauchy-Schwarz inequality, we get, for any $\zeta \in(0,1)$,

$$
\begin{aligned}
\int_{V_{x_{0}}(\tilde{\ell})^{\mathrm{C}}} \mid & \left.(\nabla-i \kappa H \mathbf{A}) \eta_{\ell} \psi\right|^{2} d x \\
\leq & \left(1+\kappa^{-\zeta}\right) \int_{V_{x_{0}}(\tilde{\ell})^{\mathrm{C}}}\left|\eta_{\ell}(\nabla-i \kappa H \mathbf{A}) \psi\right|^{2} d x+\left(1+\kappa^{\zeta}\right) \int_{V_{x_{0}}(\tilde{\ell})^{\mathrm{C}}}\left|\nabla \eta_{\ell}\right|^{2}|\psi|^{2} d x \\
\leq & \left(1+\kappa^{-\zeta}\right) \int_{V_{x_{0}}(\tilde{\ell})^{\mathrm{C}}}|(\nabla-i \kappa H \mathbf{A}) \psi|^{2} d x \\
& +\left(1+\kappa^{\zeta}\right) \int_{\{t(x) \leq \sigma \ell\} V_{x_{0}}(\tilde{\ell})^{\mathrm{C}}}\left|\nabla \eta_{\ell}\right|^{2}|\psi|^{2} d x+\left(1+\kappa^{\zeta}\right) \int_{\{t(x)>\sigma \ell\}}\left|\nabla \eta_{\ell}\right|^{2}|\psi|^{2} d x \\
\leq & \left(1+\kappa^{-\zeta}\right) \int_{V_{x_{0}}(\tilde{\ell})^{\mathrm{C}}}|(\nabla-i \kappa H \mathbf{A}) \psi|^{2} d x+C\left(1+\kappa^{\zeta}\right) .
\end{aligned}
$$

Here we used the properties of the function $\eta_{\ell}$, namely that $\eta_{\ell} \leq 1,\left|\nabla \eta_{\ell}\right|=\mathcal{O}\left(\sigma^{-1} \ell^{-1}\right)$ and $\left|\{t(x) \leq \sigma \ell\} \cap V_{x_{0}}(\tilde{\ell})^{\complement}\right|=\mathcal{O}\left(\sigma^{2} \ell^{2}\right)$. 
For the integral over $\{t(x)>\sigma \ell\}$, we use that $b\left|B_{0}\left(x_{0}\right)\right| \geq 1+\epsilon$, which in turn allows us to use Theorem 1.3 and prove that the integral of $|\psi|^{2}$ is exponentially small as $\kappa \rightarrow+\infty$.

Now we use that $\left|V_{x_{0}}(\tilde{\ell})^{\complement} \cap \operatorname{supp}\left(1-\eta_{\ell}\right)\right|=\mathcal{O}\left(\sigma \ell^{2}\right)$ to write

$$
\begin{aligned}
-\kappa^{2} \int_{V_{x_{0}(\tilde{\ell})^{\mathrm{C}}}}\left|\eta_{\ell} \psi\right|^{2} d x & =-\kappa^{2} \int_{V_{x_{0}(\tilde{\ell})^{\mathrm{C}}}}|\psi|^{2} d x+\kappa^{2} \int_{V_{x_{0}}(\tilde{\ell})^{\mathrm{C}}}\left(1-\eta_{\ell}^{2}\right)|\psi|^{2} d x \\
& \leq-\kappa^{2} \int_{V_{x_{0}}(\tilde{\ell})^{\mathrm{C}}}|\psi|^{2} d x+C \kappa^{2} \sigma \ell^{2} .
\end{aligned}
$$

This yields

$$
\begin{aligned}
\mathcal{E}_{2}(u, \mathbf{A}) \leq\left(1+\kappa^{-\zeta}\right) \int_{\Omega \backslash V_{x_{0}}(\tilde{\ell})}\left(|(\nabla-i \kappa H \mathbf{A}) \psi|^{2}-\kappa^{2}|\psi|^{2}+\frac{\kappa^{2}}{2}|\psi|^{4}\right) d x & \\
& +C\left(1+\kappa^{\zeta}+\kappa^{2} \sigma \ell^{2}\right)+\kappa^{2} H^{2} \int_{\Omega}\left|\operatorname{curl} \mathbf{A}-B_{0}\right|^{2} d x .
\end{aligned}
$$

Remembering the definition of $\mathcal{E}_{2}(\psi, \mathbf{A})$ in (4.29), we obtain

$$
\mathcal{E}_{2}(u, \mathbf{A}) \leq\left(1+\kappa^{-\zeta}\right) \mathcal{E}_{2}(\psi, \mathbf{A})+C\left(1+\kappa^{\zeta}+\kappa^{2} \sigma \ell^{2}\right)
$$

\section{Step 4: Upper bound of the local Ginzburg-Landau energy.}

Since $(\psi, \mathbf{A})$ is a minimizer of the functional $\mathcal{E}(\cdot, \cdot), \mathcal{E}(\psi, \mathbf{A}) \leq \mathcal{E}\left(0, \mathbf{A}_{0}\right)=0$ and

$$
\mathcal{E}(\psi, \mathbf{A}) \leq \mathcal{E}(u, \mathbf{A})=\mathcal{E}_{1}(u, \mathbf{A})+\mathcal{E}_{2}(u, \mathbf{A}) .
$$

Using that $\mathcal{E}(\psi, \mathbf{A}) \leq 0$, we get further

$$
\left(1+\kappa^{-\zeta}\right) \mathcal{E}(\psi, \mathbf{A}) \leq \mathcal{E}(u, \mathbf{A})=\mathcal{E}_{1}(u, \mathbf{A})+\mathcal{E}_{2}(u, \mathbf{A}) .
$$

By (4.29), we may write the simple identity $\mathcal{E}(\psi, \mathbf{A})=\mathcal{E}_{1}(\psi, \mathbf{A})+\mathcal{E}_{2}(\psi, \mathbf{A})$. Using (4.31), we get

$$
\left(1+\kappa^{-\zeta}\right) \mathcal{E}_{1}(\psi, \mathbf{A}) \leq \mathcal{E}_{1}(u, \mathbf{A})+C\left(1+\kappa^{\zeta}+\kappa^{2} \sigma \ell^{2}\right)
$$

Now, we use the estimate in (4.30) to write

$$
\begin{aligned}
& \mathcal{E}_{1}(\psi, \mathbf{A}) \leq 2 \kappa \ell \sqrt{\frac{1}{b\left|B_{0}\left(x_{0}\right)\right|}} E_{\text {surf }}\left(b B_{0}\left(x_{0}\right)\right) \\
& +C \kappa \ell\left(\kappa^{-\zeta}+\ell^{\alpha}+\kappa^{3} \ell^{3+\alpha}+\kappa \ell^{1+\alpha}+\sigma+(\kappa \ell)^{-1}+\kappa^{-1+\zeta} \ell^{-1}+\kappa \sigma \ell+\frac{|\ln (\ell \sqrt{\kappa H})|^{2}}{\ell^{2} \kappa^{2}}\right) .
\end{aligned}
$$

Step 5: Lower bound of the $L^{4}$-norm.

We select

with

$$
\ell=\kappa^{-\rho}, \quad \sigma=\kappa^{-\delta} \text { and } \quad \zeta=\frac{1-\rho}{2},
$$

$$
\frac{1}{1+\alpha}<\frac{3}{3+\alpha}<\rho<1 \text { and } 1-\rho<\delta<1 .
$$

In this way, we get that, the restriction $\bar{\Sigma}\left(\kappa, \kappa^{-\rho}, \kappa^{-\delta}\right)$ of

$$
\bar{\Sigma}(\kappa, \ell, \sigma):=\kappa^{-\zeta}+\ell^{\alpha}+\kappa^{3} \ell^{3+\alpha}+\kappa \ell^{1+\alpha}+\sigma+(\kappa \ell)^{-1}+\kappa^{-1+\zeta} \ell^{-1}+\kappa \sigma \ell+\frac{|\ln (\ell \sqrt{\kappa H})|^{2}}{\ell^{2} \kappa^{2}},
$$

tends to 0 as $\kappa \rightarrow+\infty$.

Consequently, we infer from (4.32),

$$
\mathcal{E}_{1}(\psi, \mathbf{A}) \leq 2 \kappa \ell \sqrt{\frac{1}{b\left|B_{0}\left(x_{0}\right)\right|}} E_{\text {surf }}\left(b B_{0}\left(x_{0}\right)\right)+C \kappa \ell \bar{\Sigma}\left(\kappa, \kappa^{-\rho}, \kappa^{-\delta}\right) .
$$


Now, let $f$ be the smooth function satisfying (4.18). Again, using the properties of $f$ and a straightforward computation as in Step 3, we have

$$
\begin{aligned}
& \mathcal{E}_{1}(f \psi, \mathbf{A}) \leq\left(1+\kappa^{-\zeta}\right) \mathcal{E}_{1}(\psi, \mathbf{A})+C\left(\kappa^{\zeta}+\kappa^{2} \sigma \ell^{2}\right), \\
& \int_{V_{x_{0}}(\tilde{\ell})} f^{2}\left(-1+\frac{1}{2} f^{2}\right)|\psi|^{4} d x \geq-\frac{1}{2} \int_{V_{x_{0}}(\ell)}|\psi|^{4} d x+C \sigma \ell^{2} .
\end{aligned}
$$

Using the lower bound of $\mathcal{E}_{1}(f \psi ; \mathbf{A})$ in (4.23), we get from (4.34) and (4.35),

$$
\left|\mathcal{E}_{1}(\psi, \mathbf{A})-2 \kappa \ell \sqrt{\frac{1}{b\left|B_{0}\left(x_{0}\right)\right|}} E_{\text {surf }}\left(b B_{0}\left(x_{0}\right)\right)\right| \leq C \kappa \ell \bar{\Sigma}\left(\kappa, \kappa^{-\rho}, \kappa^{-\delta}\right) .
$$

Remembering the definition of $\mathcal{E}_{1}(\psi, \mathbf{A})=\mathcal{E}_{1}\left(\psi, \mathbf{A} ; V_{x_{0}}((1+\sigma) \ell)\right)$, we get the statement concerning the local energy in Theorem 4.8 .

Now we return back to (4.21). Using (4.35), we write

$$
\left(1+\kappa^{-\zeta}\right) \mathcal{E}_{1}(\psi, \mathbf{A})+C\left(\kappa^{-\zeta}+\kappa^{2} \sigma \ell^{2}\right) \geq-\frac{\kappa^{2}}{2} \int_{V_{x_{0}}(\ell)}|\psi|^{4} d x-C \sigma \ell^{2} \kappa^{2} .
$$

Rearranging the terms, then using (4.34) and (4.33), we arrive at the following upper bound

$$
\frac{\kappa^{2}}{2} \int_{V_{x_{0}}(\ell)}|\psi(x)|^{4} d x \geq-2 \kappa \ell \sqrt{\frac{1}{b\left|B_{0}\left(x_{0}\right)\right|}} E_{\text {surf }}\left(b B_{0}\left(x_{0}\right)\right)+C \kappa \ell \bar{\Sigma}\left(\kappa, \kappa^{-\rho}, \kappa^{-\delta}\right) .
$$

Using the remark around (4.33), this finishes the proof of Theorem 4.8.

\section{The superconductivity Region: Proof of Theorem 1.7}

In this section, we present the proof of Theorem 1.7 devoted to the distribution of the superconductivity in the region

$$
\left\{x \in \bar{\Omega}, b\left|B_{0}(x)\right|<1\right\} \text { for the applied magnetic field } H=b \kappa .
$$

The proof follows by an analysis similar to the one in Section 4, so our presentation will be shorter here.

Remark 5.1. As $\ell \rightarrow 0_{+}$, the area of $\mathcal{W}\left(x_{0}, \ell\right)$ as introduced in (1.18) is

$$
\left|\mathcal{W}\left(x_{0}, \ell\right)\right|=4 \ell^{2} \quad \text { if } x_{0} \in \Omega
$$

and

$$
\left|\mathcal{W}\left(x_{0}, \ell\right)\right|=4 \ell^{2}+o\left(\ell^{2}\right) \quad \text { if } x_{0} \in \partial \Omega .
$$

The proof of Theorem 1.7 is presented in five steps. In the sequel, $\rho \in\left(\frac{2}{2+\alpha}, 1\right)$ and $c_{2}>c_{1}>0$ are fixed,

$$
c_{1} \kappa^{-\rho} \leq \ell \leq c_{2} \kappa^{-\rho} \text { and } \sigma=\kappa^{\frac{\rho-1}{2}} .
$$

We will refer to the condition on $\ell$ by writing $\ell \approx \kappa^{-\rho}$.

\section{Step 1. Useful estimates.}

Let $f$ be a smooth function such that

$$
f=1 \quad \text { in } \mathcal{W}\left(x_{0}, \ell\right), \quad 0 \leq f \leq 1 \text { and }|\nabla f| \leq \frac{C}{\sigma \ell} \quad \text { in } \mathcal{W}\left(x_{0},(1+\sigma) \ell\right) .
$$

As in the proof of (4.22), we have

$$
\mathcal{E}_{1}\left(f \psi, \mathbf{A} ; \mathcal{W}\left(x_{0},(1+\sigma) \ell\right)\right) \leq-\frac{\kappa^{2}}{2} \int_{\mathcal{W}\left(x_{0}, \ell\right)}|\psi(x)|^{4} d x+C \sigma^{-1} .
$$


Here $\mathcal{E}_{1}$ is introduced in (4.20). Furthermore, we have the following two estimates (cf. (4.35)):

$$
\begin{aligned}
\mathcal{E}_{1}\left(f \psi, \mathbf{A} ; \mathcal{W}\left(x_{0},(1+\sigma) \ell\right)\right) \leq\left(1+\kappa^{-\zeta}\right) \mathcal{E}_{1}\left(\psi, \mathbf{A} ; \mathcal{W}\left(x_{0},(1+\sigma) \ell\right)\right) & \\
& +C \kappa^{2} \ell^{2}\left(\sigma^{-1} \kappa^{\zeta}(\kappa \ell)^{-2}+\sigma\right)
\end{aligned}
$$

and (cf. (4.21))

$$
\begin{aligned}
\mathcal{E}_{1}\left(f \psi, \mathbf{A} ; \mathcal{W}\left(x_{0},(1+\sigma) \ell\right)\right) & \geq \kappa^{2} \int_{\mathcal{W}\left(x_{0},(1+\sigma) \ell\right)} f^{2}\left(-1+\frac{1}{2} f^{2}\right)|\psi|^{4} d x \\
& \geq-\frac{\kappa^{2}}{2} \int_{\mathcal{W}\left(x_{0}, \ell\right)}|\psi(x)|^{4} d x+C \sigma \ell^{2} \kappa^{2}
\end{aligned}
$$

where $\zeta \in(0,1)$ is a constant to be chosen later.

Step 2. The case $B_{0}\left(x_{0}\right)=0$.

The upper bound for the integral of $|\psi|^{4}$ in Theorem 1.7 is trivial since $|\psi| \leq 1$ and $g(0)=-\frac{1}{2}$.

We have the obvious inequalities

$\mathcal{E}_{1}\left(f \psi, \mathbf{A} ; \mathcal{W}\left(x_{0},(1+\sigma) \ell\right)\right) \geq \int_{\mathcal{W}\left(x_{0},(1+\sigma) \ell\right)}\left(-\kappa^{2}|f \psi|^{2}+\frac{\kappa^{2}}{2}|f \psi|^{4}\right) d x \geq-\frac{\kappa^{2}}{2} \int_{\mathcal{W}\left(x_{0},(1+\sigma) \ell\right)} d x$

Inserting this into (5.4) and selecting $\zeta=\frac{1-\rho}{2}$, we get

$$
\mathcal{E}_{1}\left(\psi, \mathbf{A} ; \mathcal{W}\left(x_{0},(1+\sigma) \ell\right)\right) \geq-C \kappa^{2} \ell^{2}\left(\sigma^{-1} \kappa^{\zeta}(\kappa \ell)^{-2}+\sigma\right)=o\left(\kappa^{2} \ell^{2}\right)
$$

since $\sigma=\kappa^{\frac{\rho-1}{2}}, \ell \approx \kappa^{-\rho}$ and $\frac{2}{2+\alpha}<\rho<1$.

Now we prove an upper bound for $\mathcal{E}_{1}\left(f \psi, \mathbf{A} ; \mathcal{W}\left(x_{0},(1+\sigma) \ell\right)\right)$. Let $\eta_{\ell}$ be a smooth function satisfying

$$
\eta_{\ell}(x)=0 \text { in } \mathcal{W}\left(x_{0},(1+\sigma) \ell\right), \quad \eta_{\ell}(x)=1 \text { in } \Omega \backslash \mathcal{W}\left(x_{0},(1+2 \sigma) \ell\right), \quad 0 \leq \eta_{\ell}(x) \leq 1 \text { in } \Omega,
$$

and

$$
\left|\nabla \eta_{\ell}(x)\right| \leq C \sigma^{-1} \ell^{-1} \quad \text { in } \Omega,
$$

for some constant $C>0$. We define the function

$$
u(x)=\exp (i \kappa H w(x)) f(x)+\eta_{\ell}(x) \psi(x),
$$

where the function $w$ is the sum of two functions $w_{1}$ and $w_{2}$ such that the two inequalities in (4.27) are satisfied in $\left.\mathcal{W}\left(x_{0},(1+\sigma) \ell\right)\right)$.

We have the obvious decomposition

$$
\mathcal{E}(u, \mathbf{A})=\mathcal{E}_{1}\left(\exp (i \kappa H w(x)) f(x), \mathbf{A} ; \mathcal{W}\left(x_{0},(1+\sigma) \ell\right)\right)+\mathcal{E}_{2}\left(\eta_{\ell}(x) \psi(x), \mathbf{A} ; \Omega \backslash \mathcal{W}\left(x_{0},(1+\sigma) \ell\right)\right),
$$

where $\mathcal{E}_{1}$ and $\mathcal{E}_{2}$ are introduced in (4.20).

We estimate $\mathcal{E}_{2}\left(\eta_{\ell}(x) \psi(x), \mathbf{A} ; \Omega \backslash \mathcal{W}\left(x_{0},(1+\sigma) \ell\right)\right)$ as we did in the proof of Theorem 4.8 (cf. Step 3 and (4.31)). In this way we get

$$
\begin{array}{r}
\mathcal{E}_{2}\left(\eta_{\ell}(x) \psi(x), \mathbf{A} ; \Omega \backslash \mathcal{W}\left(x_{0},(1+\sigma) \ell\right)\right) \leq\left(1+\kappa^{-\zeta}\right) \mathcal{E}_{2}\left(\psi(x), \mathbf{A} ; \Omega \backslash \mathcal{W}\left(x_{0},(1+\sigma) \ell\right)\right) \\
+C\left(\sigma^{-1} \kappa^{\zeta}+\sigma \kappa^{2} \ell^{2}\right)
\end{array}
$$


For the term $\mathcal{E}_{1}\left(\exp (i \kappa H w(x)) f(x), \mathbf{A} ; \mathcal{W}\left(x_{0},(1+\sigma) \ell\right)\right)$, we argue as in the proof of Theorem 4.8 (Step 2) and write

$$
\begin{aligned}
\mathcal{E}_{1}(\exp (i \kappa H w(x)) & \left.f(x), \mathbf{A} ; \mathcal{W}\left(x_{0},(1+\sigma) \ell\right)\right) \\
\leq & \left(1+\ell^{\alpha}\right) \mathcal{E}_{1}\left(f(x), B_{0}\left(x_{0}\right) \mathbf{A}_{0} ; \mathcal{W}\left(x_{0},(1+\sigma) \ell\right)\right)+C\left(\kappa^{2} \ell^{2+\alpha}+\kappa^{4} \ell^{4+\alpha}\right)
\end{aligned}
$$

Note that

$$
\begin{aligned}
\mathcal{E}_{1}\left(f(x), B_{0}\left(x_{0}\right) \mathbf{A}_{0} ; \mathcal{W}\left(x_{0},(1+\sigma) \ell\right)\right) & =\mathcal{E}_{1}\left(f(x), 0 ; \mathcal{W}\left(x_{0},(1+\sigma) \ell\right)\right) \\
& \leq C \sigma^{-1}+\kappa^{2} \int_{\mathcal{W}\left(x_{0},(1+\sigma) \ell\right)} f^{2}\left(-1+\frac{f^{2}}{2}\right) d x \\
& \leq C \sigma^{-1}-\frac{\kappa^{2}}{2}\left|\mathcal{W}\left(x_{0},(1+\sigma) \ell\right)\right|+C \sigma \kappa^{2} \ell^{2} .
\end{aligned}
$$

Therefore, we get the estimate

$$
\begin{aligned}
\mathcal{E}_{1}\left(\exp (i \kappa H w(x)) f(x), \mathbf{A} ; \mathcal{W}\left(x_{0},(1+\sigma) \ell\right)\right) \leq- & \left(1+\ell^{\alpha}\right) \frac{\kappa^{2}}{2}\left|\mathcal{W}\left(x_{0},(1+\sigma) \ell\right)\right| \\
& +C \kappa^{2} \ell^{2}\left(\ell^{\alpha}+\kappa^{2} \ell^{2+\alpha}+\sigma^{-1}(\kappa \ell)^{-2}+\sigma\right),
\end{aligned}
$$

and consequently

$$
\begin{aligned}
\mathcal{E}(u, \mathbf{A}) \leq-\frac{\kappa^{2}}{2}\left|\mathcal{W}\left(x_{0},(1+\sigma) \ell\right)\right|+\left(1+\kappa^{-\zeta}\right) \mathcal{E}_{2}( & \psi \\
& +C(x), \mathbf{A} ; \Omega \backslash \mathcal{W}\left(x_{0} \ell^{2}\left(\ell^{\alpha}+\kappa^{2} \ell^{2+\alpha}+\sigma^{-1} \kappa^{\zeta}(\kappa \ell)^{-2}+\sigma\right) .\right.
\end{aligned}
$$

Using that $\mathcal{E}(\psi, \mathbf{A}) \leq \min (0, \mathcal{E}(\psi, \mathbf{A}))$, we get

$$
\begin{aligned}
\left(1+\kappa^{-\zeta}\right) \mathcal{E}_{1}\left(\psi, \mathbf{A} ; \mathcal{W}\left(x_{0},(1+\sigma) \ell\right)\right) \leq-\frac{\kappa^{2}}{2} & \left|\mathcal{W}\left(x_{0},(1+\sigma) \ell\right)\right| \\
& +C \kappa^{2} \ell^{2}\left(\ell^{\alpha}+\kappa^{2} \ell^{2+\alpha}+\sigma^{-1} \kappa^{\zeta}(\kappa \ell)^{-2}+\sigma\right) .
\end{aligned}
$$

We insert this into (5.4), then we substitute the resulting inequality into (5.5). In this way we get

$$
\int_{\mathcal{W}\left(x_{0}, \ell\right)}|\psi|^{4} d x \geq \frac{1}{2}\left|\mathcal{W}\left(x_{0},(1+\sigma) \ell\right)\right|-C\left(\sigma+\sigma^{-1} \kappa^{\zeta}(\kappa \ell)^{-2}+\kappa^{2} \ell^{2+\alpha}+\ell^{\alpha}+\kappa^{-\zeta}\right) .
$$

The assumption on $\sigma$ and $\ell$ in (5.1) and the choice $\zeta=\frac{1-\rho}{2}$ yield that the term on the right hand side above is $o(1)$, hence we get the lower bound for the integral of $|\psi|^{4}$ in Theorem 1.7. Now, the estimate of the energy follows by collecting the estimates in (5.9) and (5.5).

Step 3. The case $\left|B_{0}\left(x_{0}\right)\right|>0$ : Upper bound.

We use (2.13) and (2.14) with $\gamma=\delta=\alpha$. We obtain, for some $C^{2, \alpha}$ real-valued function $w$,

$$
\begin{array}{r}
\mathcal{E}_{1}\left(f \psi, \mathbf{A} ; \mathcal{W}\left(x_{0},(1+\sigma) \ell\right)\right) \geq\left(1-\ell^{\alpha}\right) \mathcal{E}_{1}\left(e^{-i \kappa H w} f \psi, \mathbf{A}_{0} ; \mathcal{W}\left(x_{0},(1+\sigma) \ell\right)\right) \\
-C \kappa^{2} \ell^{2}\left(\ell^{\alpha}+\kappa^{2} \ell^{2+\alpha}\right) .
\end{array}
$$

If $x_{0} \in \Omega$, we get by re-scaling and (2.6) that

$$
\mathcal{E}_{1}\left(e^{-i \kappa H w} f \psi, \mathbf{A}_{0} ; \mathcal{W}\left(x_{0},(1+\sigma) \ell\right)\right) \geq 4 \kappa^{2}(1+\sigma)^{2} \ell^{2} g\left(b\left|B_{0}\left(x_{0}\right)\right|\right) .
$$


If $x_{0} \in \partial \Omega$, then we may write a lower bound for $\mathcal{E}_{1}\left(f \psi, \mathbf{A}_{0} ; \mathcal{W}\left(x_{0},(1+\sigma) \ell\right)\right)$ by converting to boundary coordinates as in Lemma 4.4 and get

$$
\begin{aligned}
\mathcal{E}_{1}\left(e^{-i \kappa H w} f \psi, \mathbf{A}_{0} ;\right. & \left.\mathcal{W}\left(x_{0},(1+\sigma) \ell\right)\right) \\
& \geq \frac{(1-C \ell)}{b\left|B_{0}\left(x_{0}\right)\right|} e^{N}\left(b\left|B_{0}\left(x_{0}\right)\right|, 2(1+\sigma) \ell \sqrt{\left|B_{0}\left(x_{0}\right)\right| \kappa H}\right)-C \kappa^{2} \ell^{2}\left(\ell+\kappa^{2} \ell^{3}\right) \\
& \geq 4 \kappa^{2}(1+\sigma)^{2} \ell^{2} g\left(b\left|B_{0}\left(x_{0}\right)\right|\right)-C \kappa^{2} \ell^{2}\left(\ell+\kappa^{2} \ell^{3}+(\kappa \ell)^{-1}\right) .
\end{aligned}
$$

Thus, we infer from (5.10), for $x_{0} \in \bar{\Omega}$,

$$
\mathcal{E}_{1}\left(f \psi, \mathbf{A} ; \mathcal{W}\left(x_{0},(1+\sigma) \ell\right)\right) \geq 4 \kappa^{2}(1+\sigma)^{2} \ell^{2} g\left(b\left|B_{0}\left(x_{0}\right)\right|\right)-C \kappa^{2} \ell^{2}\left(\ell^{\alpha}+\kappa^{2} \ell^{2+\alpha}+(\kappa \ell)^{-1}\right) .
$$

Inserting this into (5.3), we get

$$
\frac{1}{2} \int_{\mathcal{W}\left(x_{0}, \ell\right)}|\psi(x)|^{4} d x \leq 4(1+\sigma)^{2} \ell^{2} g\left(b\left|B_{0}\left(x_{0}\right)\right|\right)+C \ell^{2}\left(\ell^{\alpha}+\kappa^{2} \ell^{2+\alpha}+(\kappa \ell)^{-1}+(\kappa \ell)^{-2} \sigma^{-1}\right) .
$$

Our choice of $\sigma$ and $\ell$ in (5.1) guarantees that the term on the right side above is $o\left(\ell^{2}\right)$. Using Remark 5.1, we get the upper bound in Theorem 1.7 .

Remark 5.2. The proof in step 3 is still valid if $\left|B_{0}\left(x_{0}\right)\right| \geq \kappa^{-2 \gamma}, 0<\gamma<1-\rho$ and the following condition holds:

$$
\text { If } x_{0} \in \Omega \text { then } Q_{4 \kappa^{-\rho}}\left(x_{0}\right) \subset \Omega \text {. }
$$

Step 4. The case $\left|B_{0}\left(x_{0}\right)\right|>0$ and $x_{0} \in \partial \Omega$ : Lower bound.

For the sake of simplicity, we treat the case $B_{0}\left(x_{0}\right)>0$. The case $B_{0}\left(x_{0}\right)<0$ can be treated similarly by taking complex conjugation.

We define the function

$$
u(x)=\mathbf{1}_{\mathcal{W}\left(x_{0},(1+\sigma) \ell\right)}(x) \exp (i \kappa H w(x)) w_{R} \circ \Phi_{x_{0}}^{-1}(x)+\eta_{\ell}(x) \psi(x),
$$

where the function $\eta_{\ell}$ satisfies (5.6) and (5.7). Similarly as in (4.24), the function $w$ is the sum of two functions $w_{1}$ and $w_{2}$, defined in $\left.\mathcal{W}\left(x_{0},(1+\sigma) \ell\right)\right)$ and satisfying the two inequalities in (4.27). Finally

$$
w_{R}(s, t)=\exp \left(i \kappa H g_{x_{0}}(s, t)\right) \exp \left(\frac{-i \kappa H s t}{2}\right) u_{R}\left(s \sqrt{B_{0}\left(x_{0}\right) \kappa H}, t \sqrt{B_{0}\left(x_{0}\right) \kappa H}\right),
$$

and $g_{x_{0}}$ is the function satisfying (4.25) in $\mathcal{W}\left(x_{0}, \ell\right)$ (by Lemma 4.3). The function $u_{R} \in H_{0}^{1}\left(Q_{R}\right)$ is a minimizer of the energy $e^{D}\left(b B_{0}\left(x_{0}\right), R\right)$ for $R=2(1+\sigma) \sqrt{B_{0}\left(x_{0}\right) \kappa H}$ (cf. (2.3)). We can estimate $\mathcal{E}(u, \mathbf{A})$ similarly as we did in the proof of Theorem 4.8 and get

$$
\begin{aligned}
\mathcal{E}(u, \mathbf{A}) \leq 4(1+\sigma)^{2} \ell^{2} \kappa^{2} g\left(b B_{0}\left(x_{0}\right)\right)+\left(1+\kappa^{-\zeta}\right) & \mathcal{E}_{2}(\psi, \mathbf{A}) \\
& +C \kappa^{2} \ell^{2}\left(\ell^{\alpha}+\kappa^{2} \ell^{2+\alpha}+\sigma+\sigma^{-1} \kappa^{\zeta}(\kappa \ell)^{-2}\right),
\end{aligned}
$$

where $\zeta \in(0,1)$ will be chosen later and

$$
\begin{aligned}
\mathcal{E}_{2}(\psi, \mathbf{A})=\int_{\Omega \backslash \mathcal{W}\left(x_{0},(1+\sigma) \ell\right)}\left(|(\nabla-i \kappa H \mathbf{A}) \psi|^{2}-\kappa^{2}|\psi|^{2}+\frac{\kappa^{2}}{2}|\psi|^{4}\right) d x & \\
& +\kappa^{2} H^{2} \int_{\Omega}\left|\operatorname{curl} \mathbf{A}-B_{0}\right|^{2} d x .
\end{aligned}
$$

Now we use that $\mathcal{E}(\psi, \mathbf{A}) \leq \min (0, \mathcal{E}(u, \mathbf{A}))$ to write

$$
\begin{aligned}
\left(1+\kappa^{-\zeta}\right) \mathcal{E}_{1}\left(\psi, \mathbf{A} ; \mathcal{W}\left(x_{0},(1+\sigma) \ell\right)\right) \leq 4(1 & +\sigma)^{2} \ell^{2} \kappa^{2} g\left(b B_{0}\left(x_{0}\right)\right) \\
& +C \kappa^{2} \ell^{2}\left(\ell^{\alpha}+\kappa^{2} \ell^{2+\alpha}+\sigma+\sigma^{-1} \kappa^{\zeta}(\kappa \ell)^{-2}\right) .
\end{aligned}
$$


Now we use (5.4) and (5.5) to obtain

$$
\begin{aligned}
-\frac{\kappa^{2}}{2} \int_{\mathcal{W}\left(x_{0}, \ell\right)}|\psi(x)|^{4} d x+C \sigma \ell^{2} \kappa^{2} \leq 4\left(1+\kappa^{-\zeta}\right) & (1+\sigma)^{2} \ell^{2} \kappa^{2} g\left(b B_{0}\left(x_{0}\right)\right) \\
& +C \kappa^{2} \ell^{2}\left(\ell^{\alpha}+\kappa^{2} \ell^{2+\alpha}+\sigma+\sigma^{-1} \kappa^{-\zeta}(\kappa \ell)^{-2}\right) .
\end{aligned}
$$

We select $\zeta=\frac{1-\rho}{2}$. Remembering that $\sigma=\kappa^{\frac{\rho-1}{2}}$ and $\ell \approx \kappa^{-\rho}$ (cf. (5.1)), we get the lower bound for the integral of $|\psi|^{4}$ as in Theorem 1.7.

For the estimate of the local energy $\mathcal{E}_{1}\left(\psi, \mathbf{A} ; \mathcal{W}\left(x_{0},(1+\sigma) \ell\right)\right)$, we collect the inequalities in (5.11), (5.4), (5.5) and the lower and upper bounds for the integral of $|\psi|^{4}$.

Remark 5.3. Remark 5.2 holds for Step 4 as well.

Step 5. The case $\left|B_{0}\left(x_{0}\right)\right|>0$ and $x_{0} \in \Omega$ : Lower bound.

In this case $\mathcal{W}_{x_{0}}((1+\sigma) \ell)=Q_{2(1+\sigma) \ell}\left(x_{0}\right)$. We define the following trial state

$$
u(x)=\mathbf{1}_{\mathcal{W}\left(x_{0},(1+\sigma) \ell\right)}(x) \exp (i \kappa H w(x)) w_{R}(x)+\eta_{\ell}(x) \psi(x),
$$

where the functions $w$ and $\eta_{\ell}$ are as in Step 4,

$$
w_{R}(s, t)=\left\{\begin{array}{l}
u_{R}\left(\sqrt{B_{0}\left(x_{0}\right) \kappa H}\left(x-x_{0}\right)\right) \text { if } B_{0}\left(x_{0}\right)>0, \\
\overline{u_{R}\left(\sqrt{B_{0}\left(x_{0}\right) \kappa H}\left(x-x_{0}\right)\right)} \text { if } B_{0}\left(x_{0}\right)<0,
\end{array}\right.
$$

and $u_{R} \in H_{0}^{1}\left(Q_{R}\right)$ is a minimizer of the energy $e^{D}\left(b B_{0}\left(x_{0}\right), R\right)$ for $R=2(1+\sigma) \sqrt{B_{0}\left(x_{0}\right) \kappa H}$ (cf. $(2.3))$.

We argue as in Step 4 and obtain the lower bound for the integral of $|\psi|^{4}$ in Theorem 1.7. The details are omitted.

Acknowledgements. A.K. acknowledges financial support through a fund from Lebanese University.This work was achieved when the first author was distinguished visitor in AUB ... To be completed

\section{REFERENCES}

[1] A. Aftalion, S. Serfaty. Lowest Landau level approach for the Abrikosov lattice close to the second critical field. Selecta Math. 2,13, (2007).

[2] Y. Almog. Non-linear surface superconductivity in three dimensions in the large $\kappa$ limit. Commun. Contemp. Math. 6 (4), 637-652 (2004).

[3] Y. Almog, B. Helffer. The distribution of surface superconductivity along the boundary: on a conjecture of X. B. Pan. SIAM J. Math. Anal. 38, 1715-1732 (2007).

[4] Y. Almog, B. Helffer. Global stability of the normal state of superconductors in the presence of a strong electric current. Comm. Math. Phys. 330, 1021-1094 (2014).

[5] Y. Almog, B. Helffer, X.B. Pan. Mixed normal-superconducting states in the presence of strong electric currents. arXiv:1505.06322\%.

[6] W. Assaad, A. Kachmar. The influence of magnetic steps on bulk superconductivity. Discrete and Continuous Dynamical Systems, Series A. (In press).

[7] K. Attar. The ground state energy of the two dimensional Ginzburg-Landau functional with variable magnetic field. Annales de l'Institut Henri Poincaré - Analyse Non-Linéaire 32, 325-345 (2015).

[8] K. Attar. Energy and vorticity of the Ginzburg-Landau model with variable magnetic field. Asymptotic Analysis 93, 75-114 (2015).

[9] K. Attar. Pinning with a variable magnetic field of the two dimensional Ginzburg-Landau model. To appear in Non-linear Analysis: Theory Methods and Applications (2015).

[10] V. Bonnaillie-Noël, S. Fournais. Superconductivity in domains with corners. Rev. Math. Phys. 19 (2007), pp. 607-637.

[11] A. Contreras, X. Lamy. Persistence of superconductivity in thin shells beyond $H_{c 1}$. arXiv:1411.1078 (2014).

[12] M. Correggi, N. Rougerie. On the Ginzburg-Landau functional in the surface superconductivity regime. Commun. Math. Phys. 332, 1297-1343 (2014). 
[13] N. Dombrowski, N. Raymond. Semi-classical analysis with vanishing magnetic fields. Journal of Spectral Theory. 3, 423-464 (2013).

[14] S. Fournais, B. Helffer. Energy asymptotics for type II superconductors. Calc. Var. Partial Differential Equations 24 (3), 341-376 (2005).

[15] S. Fournais, B. Helffer. Spectral Methods in Surface Superconductivity. Progress in Nonlinear Differential Equations and Their Applications 77 Birkhäuser (2010).

[16] S. Fournais, A. Kachmar. Nucleation of bulk superconductivity close to critical magnetic field. Adv. Math. 226, 1213-1258 (2011).

[17] S. Fournais, A. Kachmar. The ground state energy of the three dimensional Ginzburg-Landau functional. Part I. Bulk regime. Communications in Partial Differential Equations 38, 339-383 (2013).

[18] S. Fournais, B. Helffer, M. Persson. Superconductivity between Hc2 and Hc3. J. Spectr. Theory 1, 273-298 (2011).

[19] S. Fournais, A. Kachmar, and M. Persson. The ground state energy of the three dimensional GinzburgLandau functional. Part II. Surface regime. J. Math. Pures Appl. 99, 343-374 (2013).

[20] B. Helffer, A. Mohamed. Semiclassical analysis for the ground state energy of a Schrödinger operator with magnetic wells. J. Funct. Anal. 138 (1), 40-81 (1996).

[21] B. Helffer, A. Morame. Magnetic bottles in superconductivity. J. Funct. Anal. 185 (2), 604-680 (2001).

[22] B. Helffer, A. Kachmar. The Ginzburg-Landau functional with a vanishing magnetic field. Arch. Ration. Mech. Anal. 218 (1), 55-122 (2015).

[23] B. Helffer, A. Kachmar. From constant to non-degenerately vanishing magnetic fields in superconductivity. To appear in Ann. Institut Henri Poincaré (Section Analyse non-linéaire) (2015).

[24] B. Helffer, Y.A. Kordyukov. Spectral gaps for periodic Schrödinger operators with hypersurface magnetic wells: Analysis near the bottom. J. Funct. Anal. 257 (10), 3043-3081 (2009).

[25] B. Helffer and X-B. Pan. Upper critical field and location of surface nucleation of superconductivity. Ann. Institut Henri Poincaré (Section Analyse Non-Linéaire) 20 (1), 145-181 (2003).

[26] A. Kachmar. A new formula for the energy of bulk superconductivity. Canadian Mathematical Bulletin. doi:10.4153/CMB-2016-004-x (in press).

[27] A. Kachmar, M. Nassrallah. The distribution of 3D superconductivity near the second critical field. arXiv:1511.08565.

[28] A. Kachmar. The Ginzburg-Landau order parameter near the second critical field. SIAM J. Math. Anal. 46 (1), 572-587 (2014).

[29] A. Kachmar. The ground state energy of the three-dimensional Ginzburg-Landau model in the mixed phase. J. Funct. Anal. 261 3328-3344 (2011).

[30] K. Lu, X.B. Pan. Eigenvalue problems of Ginzburg-Landau operator in bounded domains. Journal of Mathematical Physics 40 (6) (1999), 2647-2670.

[31] K. Lu and X-B. Pan. Estimates of the upper critical field for the Ginzburg-Landau equations of superconductivity. Physica D 127, 73-104 (1999).

[32] R. Montgomery. Hearing the zero locus of a magnetic field. Commun. Math. Phys. 168 (3), 651-675 (1995).

[33] J-P. Miqueu. Equation de Schrödinger avec un champ magnétique qui s'annule. Thèse de doctorat (in preparation).

[34] X.B. Pan. Surface superconductivity in applied magnetic fields above $H_{C_{2}}$. Commun. Math. Phys. 228, 228-370 (2002).

[35] X.B. Pan, K.H. Kwek. Schrödinger operators with non-degenerately vanishing magnetic fields in bounded domains. Trans. Amer. Math. Soc. 354 (10), 4201-4227 (2002).

[36] N. Raymond. Sharp asymptotics for the Neumann Laplacian with variable magnetic field : case of dimension 2. Annales Henri Poincaré. 10 (1), 95-122 (2009).

[37] J. Rubinstein. Six lectures on superconductivity. In: Boundaries, Interfaces, and Transitions, CRM Proceedings and Lecture Notes, Vol. 13, Providence, RI: Am. Math. Soc., 163-184 (1998).

[38] D. St. James, P.G. de Gennes. Onset of superconductivity in decreasing fields. Physics Letters 7 (5), 306-308 (1963).

[39] E. Sandier, S. Serfaty. Vortices for the Magnetic Ginzburg-Landau Model. Progress in Nonlinear Differential Equations and their Applications 70 Birkhäuser (2007).

[40] E. Sandier, S. Serfaty. The decrease of bulk superconductivity close to the second critical field in the Ginzburg-Landau model. SIAM J. Math. Anal. 34 (4), 939-956 (2003).

(B. Helffer) Laboratoire de Mathématiques Jean Leray, CnRS, Université de Nantes, France, and Laboratoire de Mathématiques, Université de Paris-Sud, Univ Paris-Saclay, France.

E-mail address: bernard.helffer@univ-nantes.fr

(A. Kachmar) Department of Mathematics, Lebanese University, Hadat, Lebanon.

E-mail address: ayman.kashmar@gmail.com 